\title{
Arabidopsis GELP7 is plasma membrane-localized acetyl xylan esterase, and its overexpression improves saccharification efficiency.
}

\section{Lavi Rastogi}

Regional Centre for Biotechnology

Aniket Anant Chaudhari

Regional Centre for Biotechnology

Raunak Sharma

Regional Centre for Biotechnology

Prashant Pawar ( $\sim$ prashant.pawar@rcb.res.in )

Regional Centre for Biotechnology https://orcid.org/0000-0002-6186-8386

\section{Research Article}

Keywords: Acetyl xylan esterase, plant cell wall, deacetylation, saccharification, GELP7, Arabidopsis, GDSL esterase

Posted Date: September 17th, 2021

DOl: https://doi.org/10.21203/rs.3.rs-847876/v1

License: (c) (i) This work is licensed under a Creative Commons Attribution 4.0 International License. Read Full License

Version of Record: A version of this preprint was published at Plant Molecular Biology on May 17th, 2022. See the published version at https://doi.org/10.1007/s11103-022-01275-8. 


\section{Abstract}

Acetyl substitution on the xylan chain is critical for stable interaction with cellulose and other cell wall polymers in the secondary cell wall. Xylan acetylation pattern is governed by Golgi and extracellular localized acetyl xylan esterase (AXE). We investigated the role of Arabidopsis clade Id from the GDSL esterase/lipase or GELP family in polysaccharide deacetylation. The investigation of the AtGELP7 T-DNA mutant line showed a decrease in stem esterase activity and an increase in stem acetyl content. We further generated overexpressor AtGELP7 transgenic lines, and these lines showed a decrease in xylan acetylation in comparison with wild type plants. Therefore, we have named this enzyme as AtAXE1. The subcellular localization studies showed that the AtAXE1 enzyme is secreted out, associated with the plasma membrane and involved in xylan de-esterification post-synthesis. The cellulose digestibility was improved in AtAXE1 overexpressor lines without pre-treatment, after alkali and xylanases pre-treatment. Furthermore, we have also established that the AtGELP7 gene is upregulated in the overexpressor line of AtMYB46, which is a secondary cell wall specific transcription factor. This transcriptional regulation can drive AtGELP7 or AtAXE1 to perform de-esterification of xylan in a tissue-specific manner.

\section{Introduction}

The plant cell wall, a complex and dynamic meshwork made up of polysaccharides, mainly cellulose, hemicellulose (xylan, xyloglucan and mannan) and pectin with lignin impregnation. It plays a pivotal role in plant growth, development, and maturation because of its flexible and rigid nature. Also, the cell wall is rich in cellulosic, non-cellulosic polysaccharides and polyphenols, a major source for the production of biofuels and other bio-based materials (Donev et al. 2018). But the complex and intricate cell wall structure is a major hurdle during the processing of plant biomass to biofuel. Enzymatic hydrolysis of the plant cell wall is significantly hindered by the presence of acetyl groups substituted on hemicellulosic and pectic polysaccharides. Also, enzymatic deacetylation of biomass lead to increase in acetate level in the fermentation medium that interferes with microbial growth by lowering the $\mathrm{pH}$ of the medium (Pawar et al. 2013). One way to deal with this problem is modulating polysaccharide acetylation by genetic manipulation of cell wall acetylation pathways in plants. In planta reduction of acetyl content exposes polysaccharides during the saccharification process. Therefore, polysaccharide acetyl esterases can be utilized to finetune the acetate level of the cell wall. In planta overexpression of fungal AXE in Arabidopsis and poplar resulted in enhanced cellulose and xylan hydrolysis. Also, decreasing xylan acetylation increases the fermentation efficiency of the Arabidopsis plant cell wall because of less accumulation of acetic acid (Pawar et al. 2016, 2017; Wang et al. 2020).

Few of the pectin acetyl esterase (PAE) and acetyl xylan esterase (AXE) are identified in plants but their role in plant cell wall organization and recycling is unclear. PAEs belong to the carbohydrate esterase (CE) family, a large family found in higher plants. Heterologous expression of poplar PtPAE1 in tobacco leads to abnormalities in floral organs (Gou et al. 2012). And overexpression of mung bean PAE in potato tubes increases the stiffness of the plant cell wall (Orfila et al. 2012). Mutation in Arabidopsis AtPAE8 and AtPAE9 reduces pectin acetylation content and inflorescence stem size (de Souza et al. 2014). Recently, 
AXE, which belongs to the GDSL esterase/lipase or GELP family have been identified and characterized in rice named as BRITTLE LEAF SHEATH 1 (BS1) and DEACETYLASE ON ARABINOSYL SIDECHAIN OF XYLAN 1 (DARX1). BS1 specifically deacetylates xylan, which is a major hemicellulose found in the secondary cell walls (Zhang et al. 2017) whereas DARX1 specifically cleaves acetyl groups present on arabinosyl residues of xylan backbone (Zhang et al. 2019). Both of these enzymes are localized in Golgi and involved in regulating the pattern of xylan acetylation. Other proteins which belong to the reduced wall acetylation (RWA) family transports acetyl-CoA or any other acetyl carrier from cytoplasm to Golgi (Pauly and Ramírez 2018). Golgi localized ALTERED XYLOGLUCAN 9 (AXY9) and TRICHOME BIREFRINGENCE-LIKE (TBL) family belong to the polysaccharide acetylation pathway. Arabidopsis axy 9 mutant showed decreased xylan and xyloglucan acetylation (Schultink et al. 2015). TBL is a multigene family and has acetyltransferase activity to specific polysaccharides. Arabidopsis $t b / 29, t b / 32$ and $t b / 33$ mutants have decreased levels of xylan acetylation (Xiong et al. 2013; Yuan et al. 2016). Golgi synthesized acetylated xylan is transported to apoplastic space and probably deacetylated by apoplastic localized AXEs. There are many uncharacterized GELPs in plants that might have a role in cell wall polysaccharide deacetylation. In Arabidopsis, no member from the GELP family having a role in cell wall polysaccharide deacetylation have been reported so far. GELP family members have a flexible enzyme active site that gives specificity to many substrates, hence contributing multiple cellular functions in plant. GELP amino acid sequence consists of five (I-V) consensus sequences and four conserved amino acid residues, i.e., Ser(S)-Gly(G)-Asn (N)-His(H) found in blocks I, II, III, and V, respectively, which are involved in catalysis. Thus, GELP is categorized in the SGNH hydrolase superfamily (Akoh et al. 2004). AtGELP33/AtFXG1 showed fucosidase activity, suggesting the GELP family role in cell wall modification (De la Torre et al. 2002). AtGELP83/AtCDEF1 and AtGELP60/AtLTL1 are involved in cutin biosynthesis and de-esterification respectively (Naranjo et al. 2006; Takahashi et al. 2010). AtGELP63/AtESM1 is involved in glucosinolate hydrolysis (Zhang et al. 2006). Rice GELPs i.e., BS1 and DARX1 are AXEs as mutation in these genes led to an increase in xylan acetylation. Rice GELP members i.e., MAO HUZI 11 (MHZ11) and RICE MALE STERILE 2 (RMS2) are involved in ethylene signaling and lipid homeostasis, respectively (Zhao et al. 2020a, b). Furthermore, IPE2 (IRREGULAR POLLEN EXINE 2) is a GDSL lipase in maize which is essential for male fertility (Huo et al. 2020). Several Arabidopsis GELP members have recently shown key roles in suberin polymerization and degradation (Ursache et al. 2021). In summary, only a few members from the GELP family are characterized, and they function in lipid, glucosinolate and cell wall metabolism by showing either lipase or esterase activity. Identification of their role in xylan deesterification will enable us to understand xylan biosynthesis, modification and recycling. And those esterases can be effectively utilized to alter plant biomass for generation of value-added products.

In this project, AtGELP7 or At1G28580 is identified as acetyl xylan esterase (AtAXE1). Transient expression of AtAXE1 in Nicotiana benthamiana revealed that it is esterase but not lipase. Arabidopsis axtaxe 1 mutant showed a decrease in esterase activity and increased stem acetyl content suggesting that this could be polysaccharide esterase. Furthermore, overexpressor transgenic lines of AtAXE1 showed a reduction in xylan acetylation. Also, an increase in cellulose and xylan digestibility in AtAXE1 
overexpressor lines revealed the potential use of decreasing polysaccharide acetylation to improve lignocellulosic biomass processing.

\section{Results}

\section{Gene members of clade Id from GELP family could be involved in xylan acetylation}

GDSL esterase/lipase or GELP family is a multigene family with diverse role in lipid and cell wall metabolism. Serine $(S)$, Glycine $(G)$, Asparagine $(N)$, and Histidine $(H)$ are conserved in four blocks in all GELP sequences. To identify acetyl xylan esterase (AXE) from the Arabidopsis GELP family, the sequences were fetched from (https://supfam.mrc-Imb.cam.ac.uk/SUPERFAMILY/) using query SGNH. A total of 103 amino acid sequences from Arabidopsis and two AXEs (BS1 and DARX1) from rice were retrieved. Neighbor-Joining tree revealed that GELP family is divided into two main clades (I and II) (Fig.S1). Clade I is divided into ten subclades (la to lj). BS1 is clubbed in clade Id, and the GDSL domain is conserved in all the sequences of clade Id. These sequences were used for phylogenetic and other bioinformatic studies. All the amino acid sequences from GELP family had GDS domain conserved at Nterminal (Fig.S2). Also, sequences from clade Id showed high sequence identity from $28-42 \%$ with rice BS1 (Fig.S3). All the members from clade Id had either TAP-like or acetylhydrolase domain. This suggested that this clade could be involved in xylan deacetylation.

\section{At GELP7 is upregulated in overexpressed MYB46 transgenic lines}

Xylan is one of the major secondary cell wall polysaccharides in dicot plants. MYB46 is a direct target of xylan biosynthetic genes. TBL29, RWA, and ATP-citrate lyase (ACL) are functioned in xylan acetylation biosynthesis and upregulated in AtSND1 overexpressor line through feedforward loop by interacting with MYB46 (Lee et al. 2011; Yuan et al. 2013; Zhong et al. 2020). Our hypothesis was GELP family members are involved in xylan acetylation and its expression is regulated by MYB46. Therefore, we generated five independent Arabidopsis transgenic lines overexpressing MYB46. All the five lines showed more expression than wild type plants (Fig. 1A). Overexpression - 2 (OE) was a highly expressed line among all with five-fold expression, followed by OE-3 and OE-5 with approximately three-fold expression. All MYB46 OE lines look like wild type plants (Fig. 1B). We found that PAL2 expression was elevated in OE-2 and OE5 lines, which was correlating with previous findings (Fig. 1C) (Yuan et al. 2013). We tested the expression of selected genes from clade Id and Ic. We did not see any change in At2G27360 (AtGELP53), At1G28670 (AtGELP14), At1G28570 (AtGELP6), At5G45910 (AtGELP10), and At1G09390 (AtGELP2). But we found that At1G28580 or AtGELP7 was by around 55-fold in both MYB46 OE lines (Fig. 1D). This suggests that AtGELP7 is likely to function in the secondary cell wall or xylan biosynthesis.

Transient expression in Nicotiana confirms that AtGELP7 or AtAXE1 is a plasma membrane-localized esterase 
AtGELP7 will be referred as AtAXE1. AtAXE1 was cloned into an expression vector having 355 constitutive promoter and transiently expressed in Nicotiana benthamiana. The members from the Arabidopsis GELP family showed both esterase and lipase activity (Lai et al. 2017). The soluble and wall-bound protein was extracted from transiently expressed AtAXE1 leaf tissue. 4-nitrophenyl acetate was used as a substrate and wall localized acetyl xylan esterase from Aspergillus niger (AnAXE1) as a positive control for esterase activity (Pawar et al. 2016). As expected, AnAXE1 showed an increase in wall-bound esterase activity (Fig. 2A). Both soluble and wall-bound protein fractions showed an increase in esterase activity where AtAXE1 was expressed in Nicotiana leaves (Fig. 2A). Some of the Arabidopsis GELP family members exhibited lipase activity (Huo et al. 2020; Zhao et al. 2020a; Ursache et al. 2021). Therefore, lipase activity was tested using 4-nitrophenyl palmitate as a substrate and similar lipase activity was observed in empty vector control, AtAXE1 and AnAXE1 expressed Nicotiana leaves (Fig. 2B). This signifies that AtAXE1 is an esterase.

To check the subcellular localization of AtAXE1 in the plant cell, AtAXE1 was fused with an N-terminal GFP tag and expressed in Nicotiana leaves along with RFP tagged plasma membrane marker. AtAXE1 GFP signal was observed in the membrane (Fig. 2C). Co-localization using RFP plasma membrane marker confirmed that it is in the plasma membrane. To distinguish GFP localization further, Nicotiana leaf epidermal cells expressing both GFP-AtAXE1 and RFP were plasmolyzed with $30 \%$ glycerol. The colocalization of both RFP and GFP signals was observed after plasmolysis. Bioinformatic analysis showed At1G28580 or AtAXE1 has a signal peptide and two transmembrane domains. (Fig.S4 and Fig.S5). Altogether this data demonstrated that AtAXE1 is a plasma membrane-localized esterase

\section{T-DNA mutant of AtAXE1 exhibited a decrease in esterase activity and an increase in polysaccharide acetylation.}

To further test the function of AtAXE1, the SALK_030805 T-DNA insertion line was obtained from Arabidopsis Biological Research Centre (ABRC). The T-DNA insertion was found at the $3^{\prime}$ region of AtAXE1 gene, suggesting that this could be a knockdown mutant (Fig. 3A). Homozygous mutants were isolated by genotyping, which morphologically look similar to the wild type plant (Fig. 3B and Fig. 3C). The RNA level expression of AtAXE1 gene was checked in leaf and stem tissues of homozygous ataxe 1 mutant by RT-PCR which showed reduction in expression of both the tissues. Mutant stem and leaf showed $82 \%$ and $37 \%$ decrease in expression in comparison with wild type plant respectively (Fig. 3D and Fig. 3E). This suggests that the SALK_030805 line is a knockdown mutant of AtAXE1. Specific esterase activity was found to be increased in mutant leaf tissue, but no significant difference in stem tissue was observed as compared to the wild-type plant (Fig.S6B and Fig.S6C). Based on bioinformatics analysis, our hypothesis was AtAXE1 could be a polysaccharide esterase. Therefore, total cell wall acetyl content was measured in leaf and stem. The acetyl content was unchanged in the leaf but increased in the stem by $12 \%$ in ataxe 1 Arabidopsis mutant (Fig. 3F and Fig. 3G). To further check relative acetylation level, dried leaf and stem powder was subjected to Fourier Transform Infrared Spectroscopy (FTIR). Comparison of wild type and mutant powder suggested an increase in acetyl linkages at $1240 \mathrm{~cm}^{-1}$ in mutant plant stem tissue but not in leaf tissue (Fig.S6D and Fig.S6E). The wavenumber $1240 \mathrm{~cm}^{-1}$ corresponds to C-O linkage in 
acetyl groups (Gorzsás et al. 2011). These results signify a relative increase in acetylation of the stem tissue. Decrease in esterase activity led to an increase in polysaccharide acetylation in ataxe 1 mutant.

\section{Analysis of Arabidopsis 35S::AtAXE1 transgenic lines further confirms that it is a wall-bound esterase}

AtAXE1 is ubiquitously expressed in all the tissues but highly in inflorescence stem, fresh rosette leaf and senescence leaf (Fig. 4A). To validate the function of AtAXE1 further, Arabidopsis transgenic lines overexpressing AtAXE1 were generated, and three independent lines, i.e., 2b, 3b, and 5b, were analyzed for further studies. Wild-type and transgenic plants had no substantial morphological distinction (Fig. 4B). Modifying polysaccharide acetylation can lead to abnormality in xylem cell morphology. It was checked by staining Arabidopsis inflorescence stem sections using toluidine blue, and no abnormalities were observed in xylem vessel cells (Fig.S7). Overexpression of the AtAXE1 gene was observed in leaf and stem tissue of transgenic lines by RT-PCR where $5 \mathrm{~b}$ line showed the highest expression in both tissues in comparison with lines $2 \mathrm{~b}$ and $3 \mathrm{~b}$ (Fig. $4 \mathrm{C}$ and Fig. 4D). Esterase activity was checked using a synthetic substrate, i.e., para-nitrophenyl acetate. It was significantly increased in both soluble and wall-bound protein fractions from leaf and stem tissues of transgenic plants (Fig. 4E and Fig. 4F).

To elucidate cell wall acetate level of stem and leaf, alcohol insoluble residues (AIR) was subjected to saponification, and acetic acid release was analyzed. The highly expressed transgenic line $5 \mathrm{~b}$ showed $16 \%$, and $2 \mathrm{~b}$, $3 \mathrm{~b}$ lines showed a $10.5 \%$ reduction in leaf acetyl content (Fig. $4 \mathrm{G}$ ). Stem acetyl content was also reduced in all transgenic lines with an average of $13 \%$ (Fig. $4 \mathrm{H})$. These results demonstrated that AtAXE1 could be involved in polysaccharide deacetylation.

\section{Sequential extraction and FT-IR analysis revealed that AtAXE1 is acetyl xylan esterase}

To investigate further into more detail whether AtAXE1 acetyl esterase prefers any specific acetylated polysaccharide as a substrate, we sequentially extracted pectin, xyloglucan and xylan in leaf fraction and analyzed the acetylation level. The transgenic line $5 \mathrm{~b}$ showed increase in acetyl content of pectin (Fig. 5A). The pellet fraction containing xyloglucan and xylan was analyzed and acetyl content was reduced in that fraction of all transgenic lines (Fig. 5B). The pellet fraction was further digested by endoglucanases to remove xyloglucan and acetyl content was decreased in all transgenic lines (Fig. 5C). This confirmed that xylan acetylation is reduced in these transgenic lines. This was verified by analyzing acetylation level in DMSO rich xylan fraction, and line $2 b$ and $5 b$ showed a decrease by $9 \%$ and $21 \%$, respectively, in comparison with wild type (Fig. 5D). To confirm these results, stem powder samples were subjected to FTIR analysis. Spectral analysis showed less abundant $1210-1275 \mathrm{~cm}^{-1}$ (C-O-C) acetyl linkage in transgenic lines in comparison with wild type of both leaf and stem (Fig. 5E and Fig. 5F). The spectral range $1730-1750 \mathrm{~cm}^{-1}$ corresponding $C=0$ acetyl linkage was also reduced in stem tissues of transgenic plants (Fig. 5f). We further calculated the ratio of acetyl to xylose linkage, and it was decreased in transgenic lines as compared with wild type (Fig. 5G and Fig. 5H). In conclusion, sequential extraction and FT-IR data confirmed that AtAXE1 is acetyl xylan esterase. 
Xylanase digested AtAXE1 transgenic lines revealed an increase in xylan digestibility and confirms that it is involved in xylan deacetylation

To test accessibility of xylanases in transgenic lines, we carried out xylan oligosaccharide mass profiling (OLIMP) analysis where insoluble alcohol residues from stem tissue of transgenic and WT plant was subjected to digestion by GH11 $\beta$-endoxylanase that resulted in the release of neutral and acidic xylooligosaccharides (XOS). Neutral XOS released ranging from degree of polymerization DP2 to DP6, which are either acetylated or non-acetylated (Fig. 6A). Acidic XOS ranging from DP3 to DP5 are acetylated and substituted with either glucuronic acid or methyl glucuronic acid (Fig. 6B). In neutral XOS profile, shorter chain XOS i.e., xylobiose (Xyl2) and diacetylated xylobiose (Xyl2Ac2) were relatively elevated in transgenic lines. Whereas less abundant diacetylated xylotriose (Xyl3Ac2), tetra or penta acetylated xylotetraose were relatively decreased in transgenic lines in comparison with wild-type. Acidic XOS analysis showed similar trend that more abundance of shorter chains GlcA-X3Ac1 and (Me)GIcA-X3Ac1 and less abundance of (Me)GlcA-X4Ac2 and (Me)GlcA-X5Ac3. Generation of more number of shorter chains indicated efficient hydrolysis in AtAXE1 OE lines. These results suggest that xylan from transgenic lines was more accessible for $\mathrm{GH} 11$ xylanases because of less xylan acetylation in transgenic lines overexpressing AtAXE1. This further confirmed that AtAXE1 is an acetyl xylan esterase, and its constitutive expression enhances xylan digestibility.

\section{Improved saccharification efficiency in AtAXE1-overexpressing Arabidopsis transgenic lines}

To test the effect of AtAXE1 overexpression on saccharification efficiency of lignocellulosic biomass, alcohol insoluble residues from ball milled dried stem of transgenic and WT plants were subjected to an enzyme cocktail containing cellulase and $\beta$-glucosidase and analyzed for the glucose release by GODPOD assay. Glucose analysis without pretreatment AIR samples showed $27 \%$ increase in glucose release in highly overexpressing $5 \mathrm{~b}$ line than in WT (Fig. 7A). In another experiment, the cell wall powder was pretreated with $0.4 \mathrm{M} \mathrm{NaOH}$, which de-esterifies and dissolves hemicellulosic polysaccharides. Cellulose digestibility was again increased in the $2 \mathrm{~b}, 3 \mathrm{~b}$, and $5 \mathrm{~b}$ lines by $5 \%, 7 \%$ and $12 \%$, respectively (Fig. 7B). We also treated biomass with xylanases, analyzed the digestibility of cellulose, and glucose release was increased by $13 \%$ and $9 \%$ in line $2 \mathrm{~b}$ and $5 \mathrm{~b}$, respectively, as compared to wild type (Fig. 7C). To further analyze changes in the cell wall because of AtAXE1 overexpression, we measured cellulose, lignin and xylan content because these polymers are major contributing factors for biomass recalcitrance. The total cellulose and xylose content were similar in wild-type plants and AtAXE1 overexpressor plants (Fig. 7D and Fig. 7E). But the lignin content was reduced in all transgenic lines compared to wild-type plants (Fig. 7F). The overall conclusion from these experiments is that AtAXE1 overexpression increases cellulose digestibility.

\section{Discussion}

The biosynthetic mechanism of xylan acetylation needs attention because of its role in maintaining xylan stability and cell wall assembly (Qaseem and Wu 2020). Golgi localized proteins involved in xylan 
acetylation, biosynthesis and modification are characterized extensively in Arabidopsis. Post synthetic xylan acetylation modification is hypothesized to be controlled by apoplastic acetyl xylan esterases (AXEs) that are uncharacterized in plants. Here, we have identified and characterized plasma membrane localized AtGELP7. The functional characterization studies revealed that it is involved in xylan deacetylation. AtGELP7 or AtAXE1 expression is induced in MYB46 overexpression lines, suggesting that AtGELP7 promoter could be direct or indirect target of MYB46. AtAXE1 overexpression decreases xylan acetylation and increases Arabidopsis polysaccharide digestibility with and without pretreatment of lignocellulosic biomass.

Arabidopsis GELP family is a multigene member family, and a few members of this family are characterized which play a role in ethylene signaling, lipid homeostasis, and suberin polymerization (Dong et al. 2016; Lai et al. 2017; Huo et al. 2020; Zhao et al. 2020a, b; Ursache et al. 2021). The two rice Golgi-localized GELPs are characterized, i.e., BS1 and DARX1, which are involved in de-esterification of xylose and arabinose residues, respectively of arabinoxylan (Zhang et al. 2017, 2019). These two esterases play an important role in regulating acetylation patterns in Golgi. The Golgi synthesized acetylated xylan is deposited to the cell wall. There are many known polysaccharide modifiers such as pectin methylesterase, pectin acetyl esterase, pectate lyase, endoxylanase, endotransglycosylase, endoglucanase which are involved in cell wall remodeling and recycling (Barnes and Anderson 2018). Poplar xylan endotransglycosylase is involved in maintaining tensional stress by finetuning interaction between xylan and cellulose (Derba-Maceluch et al. 2015). It is also known that even chain substitution of acetyl on xylan is necessary for stable interaction of xylan and cellulose (Grantham et al. 2017). And changing the acetylation level and patten of substitution destabilizes interaction between xylan-cellulose, might lead to weaker cell wall. Extracellular AXEs can maintain acetylation level and can impact cellulose and xylan interactions. Here, we have characterized plasma membrane localized AXE from Clade Id from Arabidopsis GELP family (Fig.S1). Our transient expression studies demonstrated that AtAXE1 does not show any lipase activity, but esterase activity (Fig. 2A and Fig. 2B). Confocal imaging data revealed that it is co-localizing with plasma membrane marker and its localization was further confirmed by plasmolysis (Fig. 2C). The AtAXE1 was further characterized by generating transgenic lines overexpressing AtAXE1. The esterase activity was increased in transgenic lines and acetyl content was reduced in both leaf and stem tissue (Fig. 4). The acetyl content in sequentially extracted xylan rich fraction and DMSO xylan fraction was reduced by $18 \%$ and $21 \%$ in highly expressed transgenic line $5 \mathrm{~b}$ respectively (Fig. 5). This confirmed that AtAXE1 is acetyl xylan esterase. To further confirm these results, we analyzed XOS released after xylanase digestion and observed generation of shorter chain XOS and less of longer chain XOS by MALDI-TOF-MS in AtAXE1 transgenic lines (Fig. 6). These results were corroborated with our FT-IR data where acetyl to xylose ratio was lower in transgenic lines in comparison with wild type plants (Fig. 5). In conclusion, these data confirmed that AtGELP7 is acetyl xylan esterase.

Additionally, we observed compensatory increase in pectin acetylation in highly expressed line $5 \mathrm{~b}$ (Fig. 5A). Altering polysaccharide xylan acetylation often led to increase in pectin acetylation and substitution of glucuronic acid on xylan chain (Xiong et al. 2015). Our results are consistent with previous findings that cell wall acetylation mechanism is in tight homeostasis control. OE::AtAXE1 transgenic lines 
did not show any morphological or anatomical phenotype like collapsed xylem vessel which is commonly observed in dwarf cell wall mutants (Fig. 4B and Fig.S7). Around $20 \%$ reduction in xylan acetylation was tolerated by AtAXE1 overexpressor lines as we do not see any effect on plant growth or xylem cell morphology of transgenic lines. Arabidopsis ataxe 1 mutant also showed increase in stem acetylation by $12 \%$ because of less AXE activity (Fig. 3). Increase in stem acetylation led to dwarfism in rice BS1 mutants because of vessel cell spatial patterning but this was not observed in ataxe1 mutants (Zhang et al. 2017). The probable reason is $12 \%$ increase in stem acetylation tolerated by Arabidopsis. Whether more increase in stem acetylation is tolerated by Arabidopsis needs to be investigated in higher order gelp Arabidopsis mutants.

Secondary cell wall transcription machinery is investigated widely in Arabidopsis. MYB46 downstream of SND1 transcription factor which directly regulates expression of secondary cell wall biosynthetic genes (McCarthy et al. 2009). Upregulation of MYB46 enhances lignin, xylan and cellulose biosynthesis by increasing expression of genes in biosynthetic pathways and MYB46 OE lines accumulate more xylose and lignin. OE of MYB46 and SND1 specifically upregulated xylan acetylation biosynthetic genes such as ATP-citrate lyase (ACL), RWA, and TBL29 (Lee et al. 2011; Yuan et al. 2013; Zhong et al. 2020). Most of promoter of these genes have Secondary cell wall MYB Responsive Element (SMRE) sites which is target of MYB46. Since our hypothesis was GDSL family might be involved in xylan acetylation, we generated overexpressor MYB46 lines and AtAXE1 expression was increased by 55-fold as compared to wild type plants (Fig. 1). Promoter of AtAXE1 also contains SMRE4 (ACCAACC) and SMRE5 (ACCTAAT) suggesting that MYB46 can bind directly to activate AtAXE1 expression (Zhong and Ye 2012). It cannot be ruled out that MYB46 might be indirectly activating AtAXE1 through another transcription factors. These results indicated that xylan acetylation and deacetylation mechanism is directly or indirectly regulated by SCW transcription factors.

Chemical deacetylation of cell wall polysaccharides using $\mathrm{KOH}$ led to increase in sugar yield because it reduces crosslinking between cell wall polymers, but it can negatively affect xylan solubility (Kong et al. 1992). Enzymatic deacetylation prior to cellulose hydrolysis can positively affect both cellulose and xylan digestibility (Zhang et al. 2011). Also, lowering acetyl content improves fermentation efficiency as it balances $\mathrm{pH}$ in fermentation medium (Ranatunga et al. 1997). Because of these reasons it was hypothesized that in planta deacetylation is a potential strategy to improve biomass saccharification and fermentation. Previously we have shown that heterologous expression of Aspergillus niger AXE improved polysaccharide digestion with and without pretreatment in Poplar (Pawar et al. 2017). Arabidopsis AnAXE lines with $32 \%$ decrease in stem acetyl content showed around 19\% increase in stem saccharification efficiency with hot water and alkali pretreatment (Pawar et al. 2016). Poplar AnAXE1 constitutive expression led $26 \%$ increase in glucan conversion rate because of $13 \%$ reduction in cell wall acetyl content. In this project, AtAXE1 highly expressed transgenic line $5 \mathrm{~b}$ showed $12 \%$ decrease in stem acetyl content (Fig. $4 \mathrm{H}$ ) and glucose release was increased by $27 \%$ and $12 \%$ without and with alkali pretreatment in highly expressed transgenic line (Fig. 7A and Fig. 7B). The cellulose digestibility was increased by $9 \%$ after xylanase digestion and this suggests that less xylanases concentration in cocktail will be needed in hypoacetylated plants (Fig. 7C). XOS analysis pattern after xylanases digestion also 
revealed more accessible xylan of all transgenic lines as compared to wild type plants (Fig. 6). This further suggest that in planta deacetylation of xylan increases accessibility of both xylanases and cellulases. All these modifications in acetyl content were done after post-synthetic deposition of xylan. Reducing the level of xylan acetylation in Golgi by altering expression of TBL29 gene does not impact saccharification efficiency in Arabidopsis. This suggests that post-synthetic modulation in acetylation level positively impacts polysaccharide digestibility. One of the possible hypotheses is that Golgi modified acetylated xylan tightly bound cellulose during deposition in Arabidopsis. In vivo xylan labelling techniques are necessary to validate such hypothesis. Our saccharification data corroborates with previous results that post-synthetic xylan deacetylation increases the saccharification efficiency further strengthening our hypothesis that hypoacetylated plants are attractive target for improving lignocellulosic biomass properties.

In summary, we have identified plasma-membrane localized acetyl xylan esterase from GELP family i.e., AtGELP7 or AtAXE1 which is upregulated in MYB46 OE lines. Increasing AtAXE1 activity constitutively increases cellulose and xylan digestibility in Arabidopsis inflorescence stem.

\section{Materials And Methods}

\section{Generation of phylogenetic tree}

Arabidopsis 103 amino acid sequences were fetched from superfamily database (https://supfam.mrcImb.cam.ac.uk/SUPERFAMILY/) using SGNH as a query. Rice BS1 (LOC_Os02g15230.1) and DARX1 (LOC_Os05g06720.) sequences were fetched from rice genome annotation project (http://rice.plantbiology.msu.edu/). The phylogenetic tree was generated by MEGA-X tool using NeighborJoining method with 250 bootstraps.

\section{Cloning of AtGELP7 and MYB46}

AtGELP7 (AT1G28580) and MYB46 (AT5G12870) were amplified using complete attB flanking primers PCWL41, and PCWL42 and partial attB flanking primers PCWL119, and PCWL120, respectively from Arabidopsis stem cDNA (Supplementary table S1) and cloned into pDONR ${ }^{\mathrm{TM}} 207$ using the Gateway BP Clonase Il enzyme mix (11789, Invitrogen, Canada). This pENTR-AT1G28580 and pENTRAT5G12870 were further subcloned into destination vectors pCC0995 containing constitutive 35S promoter (35S::AT1G28580, 35S::AT5G12870) (Weng et al. 2010) and pSITE-2CA (35S:GFPAT1G28580) (Chakrabarty et al. 2007) containing N-terminal tagged GFP vector using the Gateway LR Clonase II enzyme mix (11791, Invitrogen, Canada). These expression clones were transformed into Agrobacterium GV3101 strain, colonies were confirmed by PCR and used for stable and transient expression in plant.

\section{Subcellular localization}


Agrobacterium containing 35S::GFP-AT1G28580 and plasma membrane marker CD3-1007 (Nelson et al. 2007) was incubated at $28^{\circ} \mathrm{C}$, the culture was centrifuged at $6000 \mathrm{rpm}$, the pellet was resuspended in a mixture of $10 \mathrm{mM}$ MES buffer (RM1128, HiMedia, India) and $10 \mathrm{mM} \mathrm{MgCl}_{2}$ (MB237, HiMedia, India) $\mathrm{pH}-$ 5.6 and diluted to 0.4 OD. $100 \mathrm{mM}$ Acetosyringone was added to the suspension and kept overnight at room temperature. The leaves of Nicotiana benthamiana were then infiltrated with the suspension (Betsuyaku and Sawa 2009) and at $2^{\text {nd }}$ or $3^{\text {rd }}$ day infiltrated leaf sections were analyzed in LEICA SP8 confocal microscope. For plasmolysis experiment, infiltrated leaf sections were dipped in $30 \%$ glycerol for 30 min under vacuum.

\section{Genotyping of T-DNA mutant}

SALK_030805 line of AT1G28580 was obtained from Arabidopsis Biological Resource Centre (ABRC) and homozygous lines were isolated by genotyping using LP(PCWL99), RP (PCWL100), and BP(PCWL29) primers (Supplementary table S1).

\section{The total RNA extraction using Trizol method and quantitative Reverse Transcription-Polymerase Chain Reaction (qRT-PCR)}

Fresh tissue was collected from Arabidopsis rosette leaf and stem and ground in liquid nitrogen. RNA was extracted using Trizol method (15596018, Invitrogen, Canada) and extracted RNA was treated with TURBO DNA-free kit (AM1907, Invitrogen, Lithuania). cDNA synthesis of the DNAse treated $1 \mathrm{mg}$ RNA samples was done using iScript cDNA Synthesis kit (1708891, BioRad, USA). Using ACTIN2 (AT3G18780) as reference gene, the expression of MYB46 (AT5G12870), PAL2 (AT3G53260), and AtGELPs from clade Id and Ic were measured using qPCR primers listed in supplementary table S1. The relative fold change was calculated by $\Delta \Delta$ Ct method.

\section{Generation of Arabidopsis transgenic lines}

The Agrobacterium culture containing 35S::AT5G12870 (MYB46)

and 35S:AT1G28580 (AtGELP7) plasmids were grown at $28^{\circ} \mathrm{C}$ for 2 days, pelleted at $6000 \mathrm{rpm}$ for $15 \mathrm{~min}$ and suspended in transformation medium consisting of $5 \%$ sucrose (GRM3063, HiMedia, India) and $0.05 \%$ silwet-77 (PCT1554, HiMedia, India) suspension. The floral dip transformation was performed as explained in (Clough and Bent 1998). When the plants were completely dried, seeds were collected and selected against glufosinate ammonium (BASTA) (C45520, Sigma-Aldrich, Switzerland). The homozygous lines from T3 generation were used for further experiments.

\section{Staining and imaging of Arabidopsis stem sections}

Wild-type and transgenic Arabidopsis stem sections were stained with Toluidine Blue O (T3260, SigmaAldrich, USA) and imaged under Nikon DS-Qi2 ECLIPSE T $i$ fluorescence microscope under 10X and 40X magnification (Pradhan Mitra and Loqué 2014).

\section{Esterase activity}


The soluble and wall bound proteins were extracted from stem and leaf tissue as explained by (Biswal et al. 2014). The total protein concentration was determined by Bradford assay. Esterase activity was performed by p-Nitrophenyl Acetate (18432, SRL, India) as substrate, incubated at $37^{\circ} \mathrm{C}$ for $2 \mathrm{~h}$, released 4-nitrophenol quantified at $400 \mathrm{~nm}$ and specific esterase activity was calculated in nmol per min per $\mathrm{mg}$ of total protein using 4-nitrophenol standard curve.

\section{Lipase activity}

The soluble and wall bound proteins were isolated from Nicotiana leaf tissue as described by (Gupta et al. 2002). Lipase activity was performed with 4-Nitrophenyl palmitate (N2752, Sigma-Aldrich, Mongolia) as substrate, incubated at $50^{\circ} \mathrm{C}$ for $5 \mathrm{~h}$, and released 4-nitrophenol quantified at $410 \mathrm{~nm}$. Then using the BSA standard curve, the specific lipase activity was calculated in nmol of product releases per min per mg of total protein using 4-nitrophenol standard curve.

\section{Preparation of Alcohol Insoluble Residue (AIR)}

The dried plant tissue was ground in Qiagen TissueLyser II, treated with $80 \%$ ethanol in 4 mM HEPES buffer (MB016, HiMedia, India) for 30 mins at $70^{\circ} \mathrm{C}$. The mixture was then centrifuged and pellet was sequentially washed with 800 ul of 70\% ethanol, chloroform:methanol (1:1) and acetone. After removing acetone, the pellet was dried overnight.

\section{Cell wall acetyl content analysis}

$1 \mathrm{mg}$ of AIR sample was saponified using $1 \mathrm{M} \mathrm{NaOH}$, and neutralized with $1 \mathrm{M} \mathrm{HCl}$, and saponified supernatant was analyzed by acetic acid kit (K-ACET, Megazyme, Ireland).

\section{Sequential extraction of pectin, xyloglucan, and xylan}

Leaf AIR was incubated with $50 \mathrm{mM}$ ammonium formate (50504, SRL, India) buffer at $37^{\circ} \mathrm{C}$ for $24 \mathrm{~h}$ in shaker dry bath. The supernatant ammonium formate fraction was collected, and pellet was digested with pectate lyase (E-PCLYAN2, Megazyme, Ireland). Both ammonium formate and pectate lyase fractions were freeze dried and the pellet was dissolved in water. After pectin extraction, the pellet containing xyloglucan and xylan was digested with endoglucanse i.e., cellulase (C1184-5KU, SigmaAldrich, USA) to remove xyloglucan and the remaining pellet rich in xylan was freeze dried. Then pectin fraction, combined xyloglucan and xylan-rich fraction, and xylan-rich fraction were analyzed for acetic acid (K-ACET, Megazyme, Ireland), galacturonic acid (K-URONIC, Megazyme, Ireland), and xylose (KXYLOSE, Megazyme, Ireland) content.

\section{DMSO extraction of xylan}

AIR samples were delignified using 1.3\% sodium hypochlorite (Q27905, Thermo Fisher Scientific, India), incubated at $75^{\circ} \mathrm{C}$ for $2 \mathrm{~h}$ with shaking in dry bath. Samples were centrifuged, pellet was washed with acetone and dried overnight. DMSO was added to above holocellulosic fraction and incubated at $60^{\circ} \mathrm{C}$ for 
$24 \mathrm{~h}$ in shaking dry bath. Supernatant collected after centrifugation was precipitated in ethanol:methanol:water (7:2:1) mixture by keeping at $4^{\circ} \mathrm{C}$ for 2 days. Precipitated xylan was centrifuged, pellet was washed with acetone and dried overnight. This DMSO extracted xylan was used for further analysis.

\section{Fourier Transform Infrared (FTIR) spectroscopy analysis}

Qiagen TissueLyser II milled dried stem powder and AIR from fresh leaf tissues were placed on ZnSe ATR crystal in Bruker Tensor27 and scanned 16 times to get the absorbance peaks of different functional groups at wavenumbers ranging from $800 \mathrm{~cm}^{-1}$ to $2000 \mathrm{~cm}^{-1}$. Acetyl linkages were detected at $1240 \mathrm{~cm}^{-1}$, and $1740 \mathrm{~cm}^{-1}$ and xylose linkages were detected at $976 \mathrm{~cm}^{-1}, 1037 \mathrm{~cm}^{-1}$, and $1073 \mathrm{~cm}^{-1}$ by analyzing birchwood xylan (partially acetylated) (P-ACXYL, Megazyme, Ireland) and beechwood xylan (P-XYLNBE, Megazyme, Ireland) as controls.

\section{XOS analysis by MALDI-TOF-MS}

$3 \mathrm{mg}$ of AIR from dried stem tissue was digested with GH11 endo-1,4- $\beta$-Xylanase (E-XYLAA, Megazyme, Ireland) at $60^{\circ} \mathrm{C}$ for 2 days and the remaining hydrolysate was purified as explained in (Chong et al. 2011). Both neutral and acidic XOS fractions were subjected to MALDI analysis separately.

\section{Cell wall composition}

Xylose content: $2 \mathrm{mg}$ of AIR sample was treated with $100 \mu \mathrm{l}$ of $1.3 \mathrm{M} \mathrm{HCL}$, incubated in dry bath at $100^{\circ} \mathrm{C}$ for $1 \mathrm{hr}$. The samples were neutralized with $100 \mu \mathrm{l}$ of $1.3 \mathrm{M} \mathrm{NaOH}$ and final volume was made up to $1 \mathrm{ml}$ by adding MilliQ water, centrifuged at $1500 \mathrm{~g}$ for 10 mins. $50 \mu \mathrm{l}$ supernatant was used for xylose analysis and analyzed by Megazyme Kit (K-XYLOSE, Megazyme, Ireland).

Cellulose content: AIR sample was digested with a-amylase (A3176, Sigma-Aldrich, USA) at $45^{\circ} \mathrm{C}$ to remove starch. Destarched cell wall residue was used to generate glucose monomer which was analyzed by anthrone assay (Updegraff 1969).

Acetyl Bromide Soluble Lignin (ABSL): AIR samples were incubated with freshly prepared $25 \%$ acetyl bromide (135968, Sigma-Aldrich, Mongolia) containing acetic acid at $50^{\circ} \mathrm{C}$ for $2 \mathrm{~h}$ The supernatant was diluted with $2 \mathrm{M} \mathrm{NaOH}$ and freshly prepared hydroxylamine hydrochloride (159417, Sigma-Aldrich,). The absorbance was taken at $280 \mathrm{~nm}$ and lignin content was represented in $\mathrm{mg}$ per $\mathrm{g}$ of AIR (Foster et al. 2010).

\section{Saccharification analysis}

Alkali pre-treatment: AIR sample was treated with $0.4 \mathrm{M} \mathrm{NaOH}$ at $90^{\circ} \mathrm{C}$ for $30 \mathrm{~min}$. The supernatant was removed by centrifugation, the pellet was washed with water and acetone and dried for further analysis. 
Xylanase pre-treatment: AIR from dried stem tissue was digested with $\mathrm{GH} 11$ endo-1,4- $\beta$-Xylanase (EXYLAA, Megazyme, Ireland) at $60^{\circ} \mathrm{C}$ for $48 \mathrm{hrs}$. The pellet was washed with acetone, dried and used for further analysis.

Preparation of enzyme mix for saccharification: Equimolar enzyme solutions $(40 \mathrm{U} / \mathrm{ml})$ were prepared for both cellulase (C1184-5KU, Sigma-Aldrich, USA) and $\beta$-glucosidase (E-BGLUC, Megazyme, Ireland). The enzyme solutions were then mixed with $0.1 \mathrm{M}$ acetic acid buffer $(\mathrm{pH}-4.8)$ in ratio 1:2 separately. The individual solution desalted through PD 10 column (17085101, GE Healthcare, UK). The desalted cellulase and $\beta$-glucosidase was then mixed in 8:2 ratio to prepare enzyme mix. Finally, the enzyme mix was diluted ten times with $0.1 \mathrm{M}$ acetic acid buffer $(\mathrm{pH}-4.8)$ to make a diluted enzyme mix for saccharification.

Enzymatic hydrolysis: The untreated or treated cell wall powder was suspended in reaction mixture containing $0.1 \mathrm{M}$ acetic acid buffer $(\mathrm{pH}-4.8)$ and enzyme mix (8:2) of cellulase and $\beta$ - glucosidase. All the samples were incubated at $50^{\circ} \mathrm{C}$ for $96 \mathrm{~h}$, centrifuged, the glucose in supernatant was analyzed by Glucose oxidase (GOD) - Peroxidase (POD) assay (Acker et al. 2016). To analyze the glucose content, the sample collected after saccharification was incubated with GOD-POD solution at $37^{\circ} \mathrm{C}$ for 30 mins. This GOD-POD mixture was prepared by mixing glucose oxidase (61788, SRL, India), Peroxidase ex. Horseradish (73292, SRL, India), 2,2'-Azino-bis (3-ethylbenzothiazoline-6-sulfonic acid) (A9941, SigmaAldrich, USA) in $0.1 \mathrm{M}$ acetic acid buffer ( $\mathrm{pH}$ 4.5). The absorbance was measured after incubation at 405 nm. Glucose content was calculated using standard curve.

\section{Statistical analysis}

The p value was calculated using Student's t-test in Office Excel 365.

\section{Declarations}

\section{Acknowledgments}

The plasmid sequencing and MALDI was performed at Advanced Technology Platform Centre (ATPC),

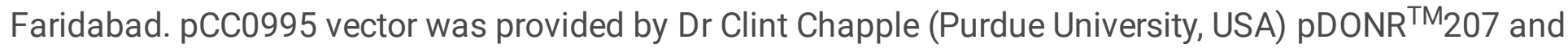
pSITE-2CA vectors were provided by Dr. Saikat Bhattacharjee (Regional Centre for Biotechnology, Faridabad). Prince Singh and Mansi Srivastav assisted us in few experiments. Suraj Tiwari provided technical assistance during confocal imaging.

\section{Funding}

This work is supported by DST-INSPIRE faculty fellowship (DST/INSPIRE/04/2018/000215) and SERBSRG Grant (SRG/2020/000861).

\section{Author contributions}


PM-AP designed the research. LR performed most of the experimental analysis. AAC performed saccharification assay. RS performed initial cloning experiment. PM-AP and LR wrote the manuscript. All authors have read and agreed to publish the manuscript.

\section{Conflicts of interest}

Authors declare no conflict of interest

\section{Consent to participate}

All authors agree

\section{Consent for publications}

All authors agree

\section{References}

1. Acker R, Vanholme R, Piens K, Boerjan W (2016) Saccharification Protocol for Small-scale Lignocellulosic Biomass Samples to Test Processing of Cellulose into Glucose. Bio-Protocol 6:1-9. https://doi.org/10.21769/bioprotoc. 1701

2. Akoh CC, Lee GC, Liaw YC et al (2004) GDSL family of serine esterases/lipases. Prog Lipid Res 43:534-552. https://doi.org/10.1016/j.plipres.2004.09.002

3. Barnes WJ, Anderson CT (2018) Release, Recycle, Rebuild: Cell-Wall Remodeling, Autodegradation, and Sugar Salvage for New Wall Biosynthesis during Plant Development. Mol Plant 11:31-46. https://doi.org/10.1016/j.molp.2017.08.011

4. Betsuyaku S, Sawa F (2009) Infiltration of. 2009:4-5

5. Biswal AK, Soeno K, Gandla ML et al (2014) Aspen pectate lyase PtxtPL1-27 mobilizes matrix polysaccharides from woody tissues and improves saccharification yield. Biotechnol Biofuels 7:113. https://doi.org/10.1186/1754-6834-7-11

6. Chakrabarty R, Banerjee R, Chung SM et al (2007) pSITE vectors for stable integration or transient expression of autofluorescent protein fusions in plants: Probing Nicotiana benthamiana-virus interactions. Mol Plant-Microbe Interact 20:740-750. https://doi.org/10.1094/MPMI-20-7-0740

7. Chong SL, Nissilä T, Ketola RA et al (2011) Feasibility of using atmospheric pressure matrix-assisted laser desorption/ionization with ion trap mass spectrometry in the analysis of acetylated xylooligosaccharides derived from hardwoods and Arabidopsis thaliana. Anal Bioanal Chem 401:2995-3009. https://doi.org/10.1007/s00216-011-5370-z

8. Clough SJ, Bent AF (1998) Floral dip: A simplified method for Agrobacterium-mediated transformation of Arabidopsis thaliana. Plant J 16:735-743. https://doi.org/10.1046/j.1365313X.1998.00343.x 
9. De la Torre F, Sampedro J, Zarra I, Revilla G (2002) AtFXG1, an Arabidopsis gene encoding a-Lfucosidase active against fucosylated xyloglucan oligosaccharides. Plant Physiol 128:247-255. https://doi.org/10.1104/pp.010508

10. de Souza A, Hull PA, Gille S, Pauly M (2014) Identification and functional characterization of the distinct plant pectin esterases PAE8 and PAE9 and their deletion mutants. Planta 240:1123-1138. https://doi.org/10.1007/s00425-014-2139-6

11. Derba-Maceluch M, Awano T, Takahashi J et al (2015) Suppression of xylan endotransglycosylase PtxtXyn10A affects cellulose microfibril angle in secondary wall in aspen wood. New Phytol 205:666-681. https://doi.org/10.1111/nph.13099

12. Donev E, Gandla ML, Jönsson LJ, Mellerowicz EJ (2018) Engineering non-cellulosic polysaccharides of wood for the biorefinery. Front Plant Sci 871:. https://doi.org/10.3389/fpls.2018.01537

13. Dong X, Yi H, Han CT et al (2016) GDSL esterase/lipase genes in Brassica rapa L.: Genome-wide identification and expression analysis. Mol Genet Genomics 291:531-542. https://doi.org/10.1007/s00438-015-1123-6

14. Foster CE, Martin TM, Pauly M (2010) Comprehensive compositional analysis of plant cell walls (Lignocellulosic biomass) part I: Lignin. J Vis Exp 5-8. https://doi.org/10.3791/1745

15. Gorzsás A, Stenlund H, Persson P et al (2011) Cell-specific chemotyping and multivariate imaging by combined FT-IR microspectroscopy and orthogonal projections to latent structures (OPLS) analysis reveals the chemical landscape of secondary xylem. Plant J 66:903-914. https://doi.org/10.1111/j.1365-313X.2011.04542.x

16. Gou JY, Miller LM, Hou G et al (2012) Acetylesterase-mediated deacetylation of pectin impairs cell elongation, pollen germination, and plant reproduction. Plant Cell 24:50-65. https://doi.org/10.1105/tpc.111.092411

17. Grantham NJ, Wurman-Rodrich J, Terrett OM et al (2017) An even pattern of xylan substitution is critical for interaction with cellulose in plant cell walls. Nat Plants 3:859-865. https://doi.org/10.1038/s41477-017-0030-8

18. Gupta N, Rathi P, Gupta R (2002) Simplified para-nitrophenyl palmitate assay for lipases and esterases. Anal Biochem 311:98-99. https://doi.org/10.1016/S0003-2697(02)00379-2

19. Huo Y, Pei Y, Tian Y et al (2020) IRREGULAR POLLEN EXINE2 encodes a gdsl lipase Essential for male fertility in maize. Plant Physiol 184:1438-1454. https://doi.org/10.1104/pp.20.00105

20. Kong F, Engler CR, Soltes EDJ (1992) Effects of Cell-Wall Acetate, Xylan Backbone, and Lignin on Enzymatic Hydrolysis of Aspen Wood. 34:23-35

21. Lai CP, Huang LM, Chen LFO et al (2017) Genome-wide analysis of GDSL-type esterases/lipases in Arabidopsis. Plant Mol Biol 95:181-197. https://doi.org/10.1007/s11103-017-0648-y

22. Lee C, Teng Q, Zhong R, Ye ZH (2011) The Four Arabidopsis REDUCED WALL ACETYLATION Genes are expressed in secondary wall-containing cells and required for the acetylation of Xylan. Plant Cell Physiol 52:1289-1301. https://doi.org/10.1093/pcp/pcr075 
23. McCarthy RL, Zhong R, Ye ZH (2009) MYB83 is a direct target of SND1 and acts redundantly with MYB46 in the regulation of secondary cell wall biosynthesis in arabidopsis. Plant Cell Physiol 50:1950-1964. https://doi.org/10.1093/pcp/pcp139

24. Naranjo M, Forment J, Roldán M et al (2006) Overexpression of Arabidopsis thaliana LTL1, a saltinduced gene encoding a GDSL-motif lipase, increases salt tolerance in yeast and transgenic plants. Plant Cell Environ 29:1890-1900. https://doi.org/10.1111/j.1365-3040.2006.01565.x

25. Nelson BK, Cai X, Nebenführ A (2007) A multicolored set of in vivo organelle markers for colocalization studies in Arabidopsis and other plants. Plant J 51:1126-1136. https://doi.org/10.1111/j.1365-313X.2007.03212.x

26. Orfila C, Degan FD, Jørgensen B et al (2012) Expression of mung bean pectin acetyl esterase in potato tubers: Effect on acetylation of cell wall polymers and tuber mechanical properties. Planta 236:185-196. https://doi.org/10.1007/s00425-012-1596-z

27. Pauly M, Ramírez V (2018) New Insights Into Wall Polysaccharide O -Acetylation. 9:1-12. https://doi.org/10.3389/fpls.2018.01210

28. Pawar PMA, Derba-Maceluch M, Chong SL et al (2016) Expression of fungal acetyl xylan esterase in Arabidopsis thaliana improves saccharification of stem lignocellulose. Plant Biotechnol J 14:387397. https://doi.org/10.1111/pbi.12393

29. Pawar PMA, Derba-Maceluch M, Chong SL et al (2017) In muro deacetylation of xylan affects lignin properties and improves saccharification of aspen wood. Biotechnol Biofuels 10:1-11. https://doi.org/10.1186/s13068-017-0782-4

30. Pawar PMA, Koutaniemi S, Tenkanen M, Mellerowicz EJ (2013) Acetylation of woody lignocellulose: Significance and regulation. Front Plant Sci 4:1-8. https://doi.org/10.3389/fpls.2013.00118

31. Pradhan Mitra P, Loqué D (2014) Histochemical staining of Arabidopsis thaliana secondary cell wall elements. J Vis Exp 1-11. https://doi.org/10.3791/51381

32. Qaseem MF, Wu AM (2020) Balanced xylan acetylation is the key regulator of plant growth and development, and cell wall structure and for industrial utilization. Int J Mol Sci 21:1-21. https://doi.org/10.3390/ijms21217875

33. Ranatunga TD, Jervis J, Helm RF et al (1997) Identification of Inhibitory Components Toxic Toward Zymomonas mobilis CP4(pZB5) Xylose Fermentation. Appl Biochem Biotechnol - Part A Enzym Eng Biotechnol 67:185-198. https://doi.org/10.1007/BF02788797

34. Schultink A, Naylor D, Dama M, Pauly M (2015) The role of the plant-specific altered xyloglucan 9 protein in arabidopsis cell wall polysaccharide o-acetylation. Plant Physiol 167:1271-1283. https://doi.org/10.1104/pp.114.256479

35. Takahashi K, Shimada T, Kondo M et al (2010) Ectopic expression of an esterase, which is a candidate for the unidentified plant cutinase, causes cuticular defects in arabidopsis thaliana. Plant Cell Physiol 51:123-131. https://doi.org/10.1093/pcp/pcp173

36. Updegraff DM (1969) Semimicro determination of cellulose inbiological materials. Anal Biochem 32:420-424. https://doi.org/10.1016/S0003-2697(69)80009-6 
37. Ursache R, De Jesus Vieira Teixeira C, Dénervaud Tendon V et al (2021) GDSL-domain proteins have key roles in suberin polymerization and degradation. Nat Plants 7:353-364. https://doi.org/10.1038/s41477-021-00862-9

38. Wang Z, Pawar PMA, Derba-Maceluch M et al (2020) Hybrid Aspen Expressing a Carbohydrate Esterase Family 5 Acetyl Xylan Esterase Under Control of a Wood-Specific Promoter Shows Improved Saccharification. Front Plant Sci 11:1-13. https://doi.org/10.3389/fpls.2020.00380

39. Weng JK, Mo H, Chapple C (2010) Over-expression of F5H in COMT-deficient Arabidopsis leads to enrichment of an unusual lignin and disruption of pollen wall formation. Plant J 64:898-911. https://doi.org/10.1111/j.1365-313X.2010.04391.x

40. Xiong G, Cheng K, Pauly M (2013) Xylan O-acetylation impacts xylem development and enzymatic recalcitrance as indicated by the arabidopsis mutant tbl29. Mol Plant 6:1373-1375. https://doi.org/10.1093/mp/sst014

41. Xiong G, Dama M, Pauly M (2015) Glucuronic Acid Moieties on Xylan Are Functionally Equivalent to O-Acetyl-Substituents. Mol Plant 8:1119-1121. https://doi.org/10.1016/j.molp.2015.02.013

42. Yuan $Y$, Teng Q, Zhong R et al (2016) Mutations of Arabidopsis TBL32 and TBL33 affect xylan acetylation and secondary wall deposition. PLoS One 11:1-24. https://doi.org/10.1371/journal.pone.0146460

43. Yuan Y, Teng Q, Zhong R, Ye ZH (2013) The arabidopsis DUF231 domain-containing protein ESK1 mediates 2-o- and 3-o-acetylation of xylosyl residues in xylan. Plant Cell Physiol 54:1186-1199. https://doi.org/10.1093/pcp/pct070

44. Zhang B, Zhang L, Li F et al (2017) Control of secondary cell wall patterning involves xylan deacetylation by a GDSL esterase. Nat Plants 3:1-9. https://doi.org/10.1038/nplants.2017.17

45. Zhang J, Siika-Aho M, Tenkanen M, Viikari L (2011) The role of acetyl xylan esterase in the solubilization of xylan and enzymatic hydrolysis of wheat straw and giant reed. Biotechnol Biofuels 4:. https://doi.org/10.1186/1754-6834-4-60

46. Zhang L, Gao C, Mentink-Vigier F et al (2019) Arabinosyl deacetylase modulates the arabinoxylan acetylation profile and secondary wall formation. Plant Cell 31:1113-1126. https://doi.org/10.1105/tpc.18.00894

47. Zhang Z, Ober JA, Kliebenstein DJ (2006) The gene controlling the quantitative trait locus EPITHIOSPECIFIER MODIFIER1 alters glucosinolate hydrolysis and insect resistance in Arabidopsis. Plant Cell 18:1524-1536. https://doi.org/10.1105/tpc.105.039602

48. Zhao H, Duan K, Li X et al (2020a) The GDSL Lipase MHZ11 Modulates Ethylene Signaling in. 32:1626-1643. https://doi.org/10.1105/tpc. 19.00840

49. Zhao J, Long T, Wang $Y$ et al (2020b) RMS2 encoding a GDSL lipase mediates lipid homeostasis in anthers to determine rice male fertility. Plant Physiol 182:2047-2064. https://doi.org/10.1104/PP.19.01487

50. Zhong R, Cui D, Richardson EA et al (2020) Cytosolic acetyl-CoA generated by ATP-citrate lyase is essential for acetylation of cell wall polysaccharides. Plant Cell Physiol 61:64-75. 
https://doi.org/10.1093/pcp/pcz178

51. Zhong R, Ye ZH (2012) MYB46 and MYB83 bind to the SMRE sites and directly activate a suite of transcription factors and secondary wall biosynthetic genes. Plant Cell Physiol 53:368-380. https://doi.org/10.1093/pcp/pcr185

\section{Figures}

(A)

Expression of MYB46

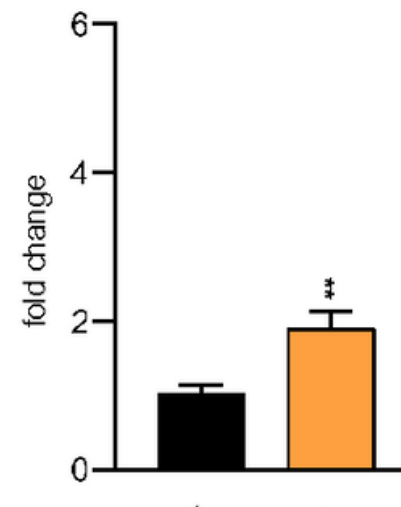

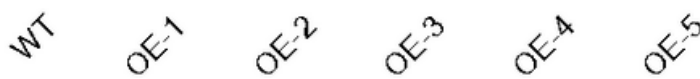

(C)

Expression of $P A L 2$

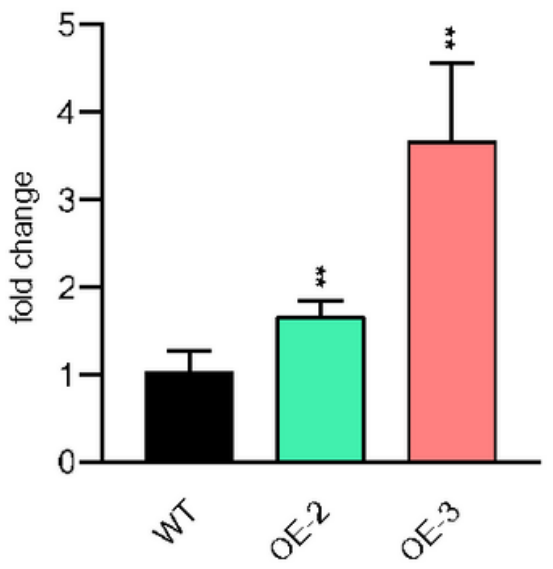

(D)

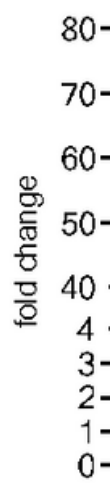

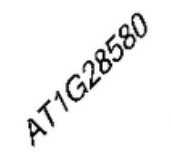

(B)

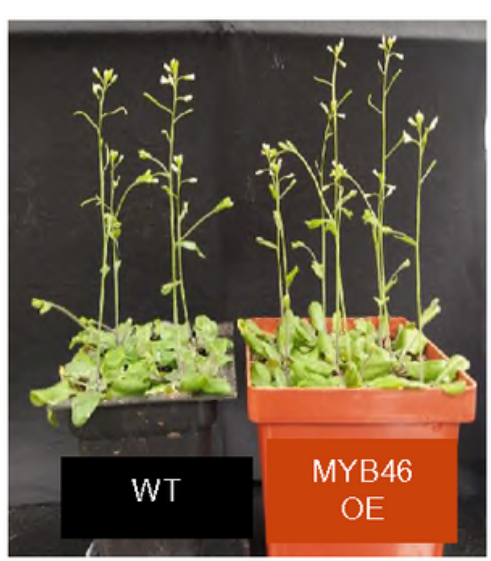

Expression of AtGELPS

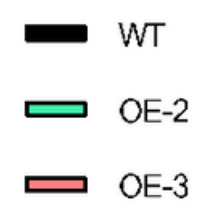

WT

एOE-2

OE-3

Figure 1 
Representation of MYB46 overexpressor line and expression of Arabidopsis GELPs in 35S::MYB46 overexpressing lines. A Expression of MYB46 in 5 independent MYB46 transgenic lines. B Representative picture of wild type and MYB46 transgenic line. C Expression of PAL2, involved in lignin biosynthetic pathway and (D) Expression of AtGELPs in 2 highly overexpressing MYB46 transgenic lines, MYB46 OE-2 and MYB46 OE-3. Data represents mean $\pm S E, n=3-4$ biological replicates, Student's t-test at ** $p \leq 0.05$, * $\mathrm{p} \leq 0.1$.

(A)

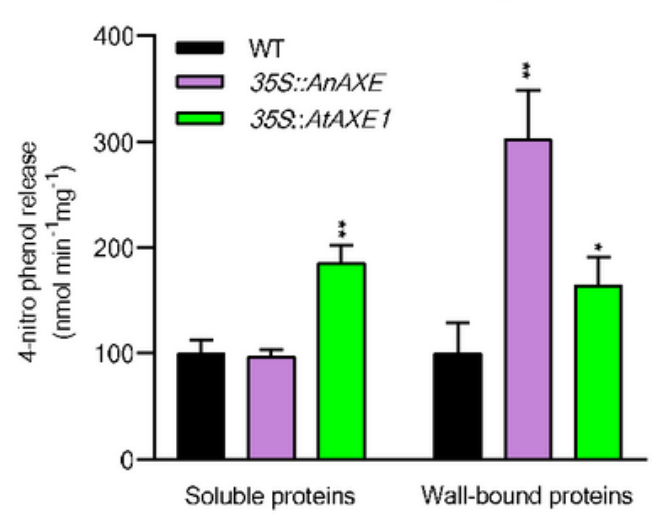

(B) Lipase activity

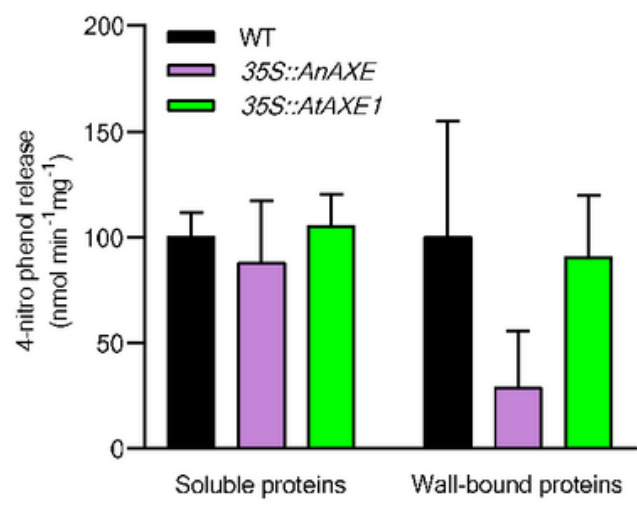

(C)

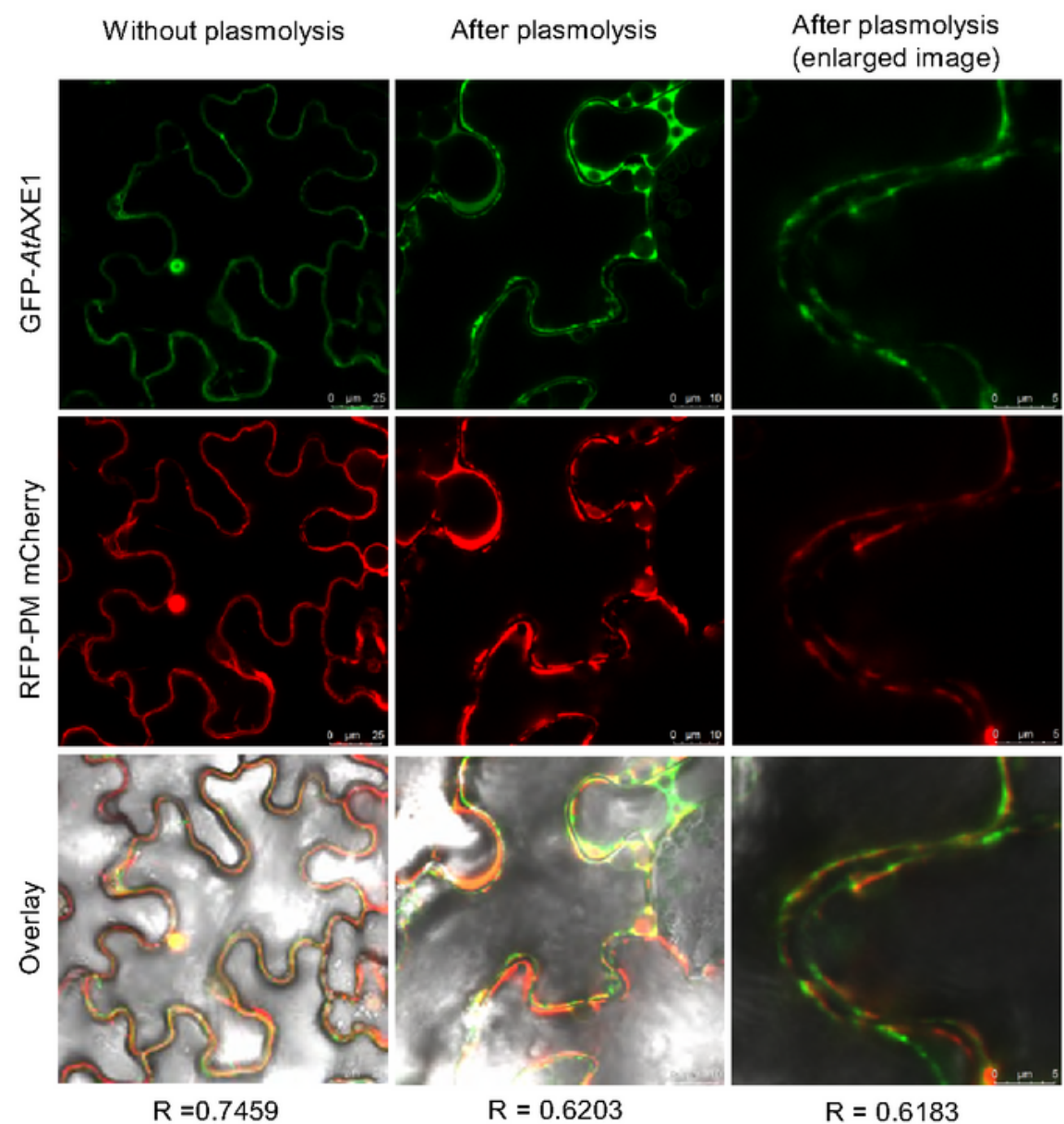

Figure 2 
Transient expression of AtAXE1 in Nicotiana leaves. Infiltrated Nicotiana benthamiana leaves with Agrobacterium carrying 35S::AtAXE1 and performed (A) esterase activity using 4-nitrophenyl acetate as a substrate, and (B) lipase activity using 4-nitrophenyl palmitate as a substrate, and product 4-nitrophenol was analysed in both assays by spectrophotometer at $400 \mathrm{~nm}$. C AtAXE1 was fused with N-terminal GFP and coinfilterated with plasma membrane RFP marker in Nicotiana leaf epidermal cells. The pictures were captured before and after plasmolysis with $30 \%$ glycerol after 2 days of infiltration. $R$ represents Pearson's Correlation. Data represents mean $\pm S E, n=3-4$ biological replicates, Student's t-test at ${ }^{*} p \leq$ $0.05,{ }^{*} \mathrm{p} \leq 0.1$. 
(A)

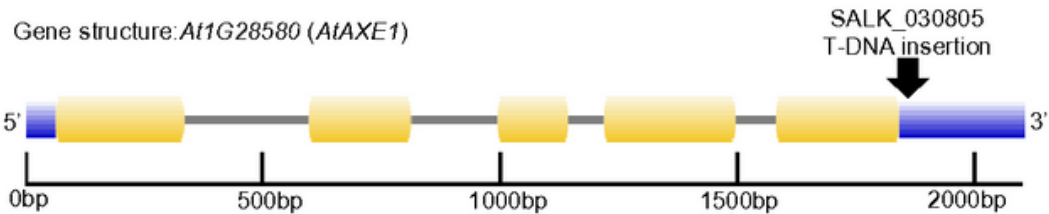

EUTR CDS

$1500 \mathrm{bp}$

2000bp

(B)

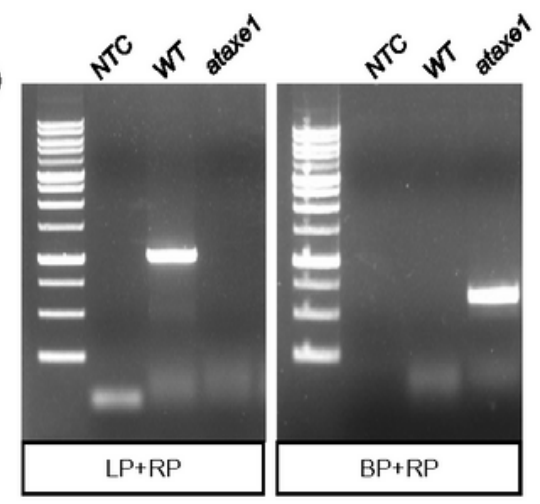

(D)

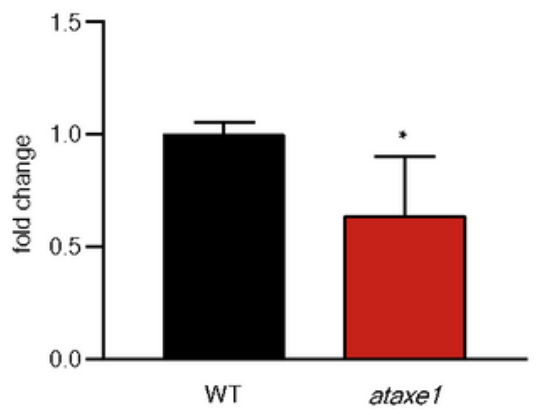

(F) Cell wall acetyl content in leaf tissue

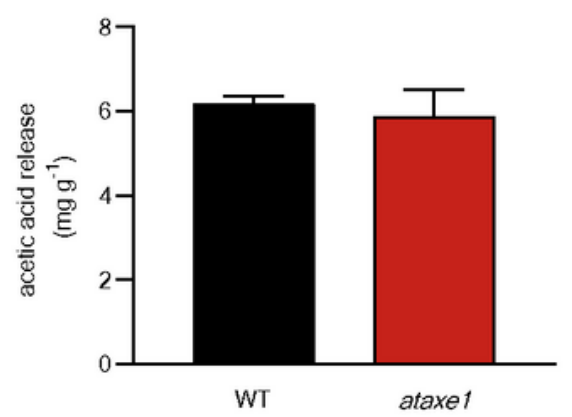

(C)

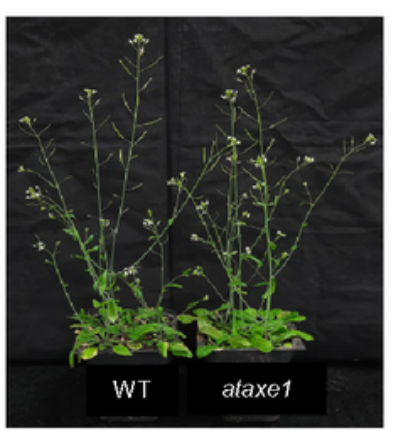

(E)

Expression in stem tissue

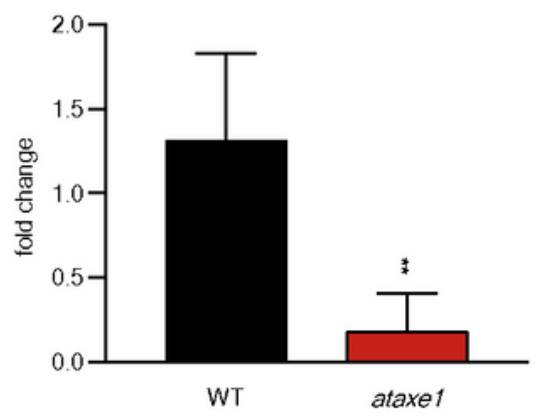

(G) Cell wall acetyl content in stem tiss

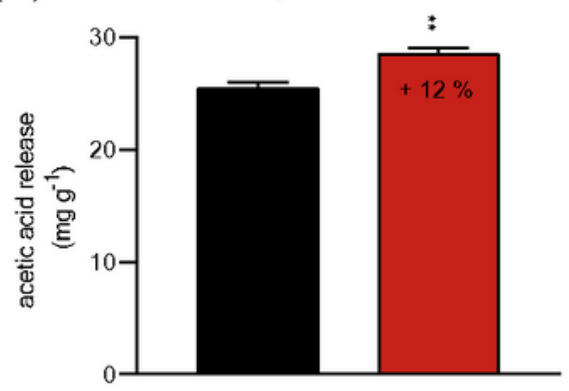

WT

ataxe1

\section{Figure 3}

Screening of T-DNA mutant line of AtAXE1, referred as ataxe1. A Gene structure of AtAXE1 showing SALK_030805 T-DNA insertion at 3' UTR. B Agarose gel showing homozygous line confirmed by LP, RP and BP primers. C Picture of 6 weeks old Arabidopsis wild type and ataxe1 mutant. RNA level expression of AtAXE1 (D) in leaf, and (E) in stem tissue of homozygous mutant plant by RT-PCR. Wild-type and mutant Arabidopsis (F) leaf, and (G) stem alcohol insoluble residues saponified and acetyl content was 
analysed by acetic acid kit (K-ACET) from Megazyme. Data represents mean $\pm \mathrm{SE}, \mathrm{n}=3-4$ biological replicates, Student's t-test at ${ }^{* *} p \leq 0.05,{ }^{*} p \leq 0.1$.

(A)

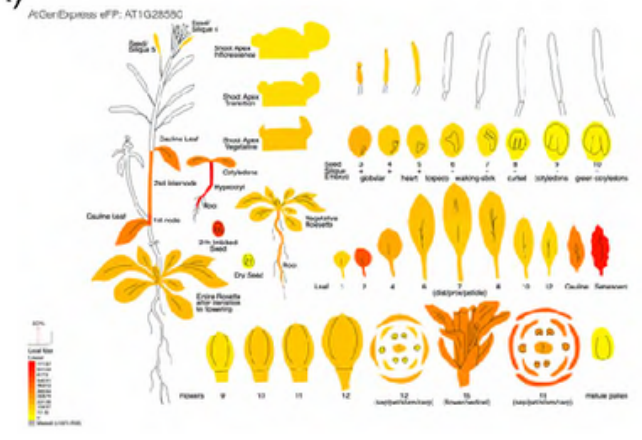

(C)

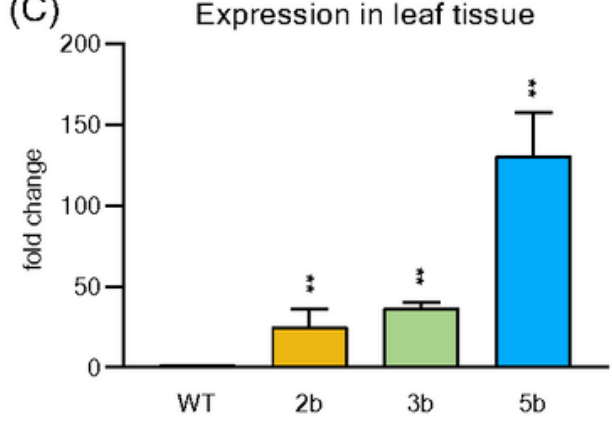

(E)

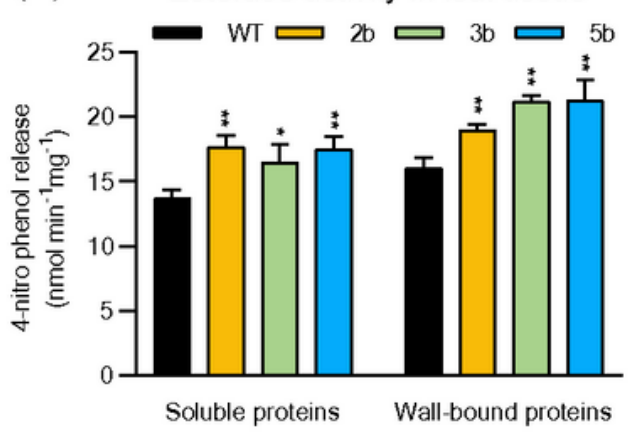

(G) Cell wall acetyl content in leaf

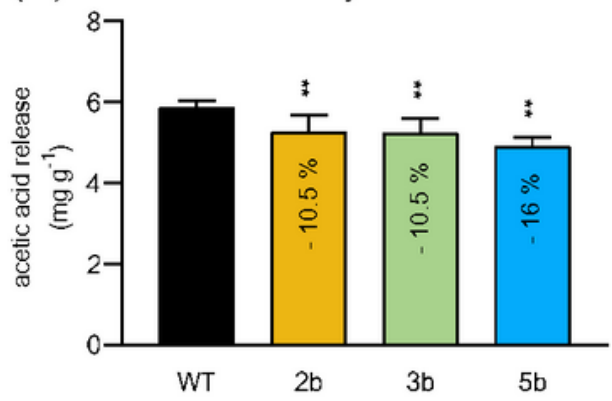

(B)

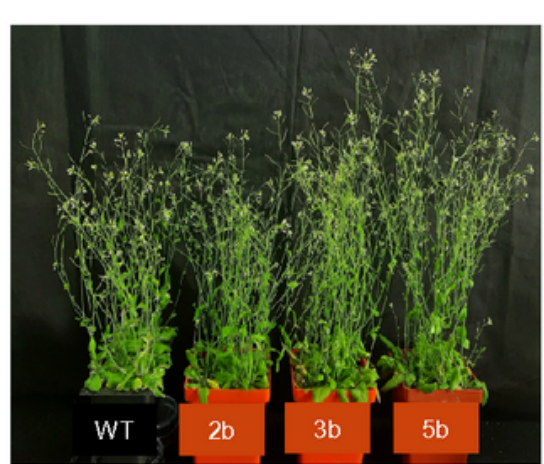

(D)

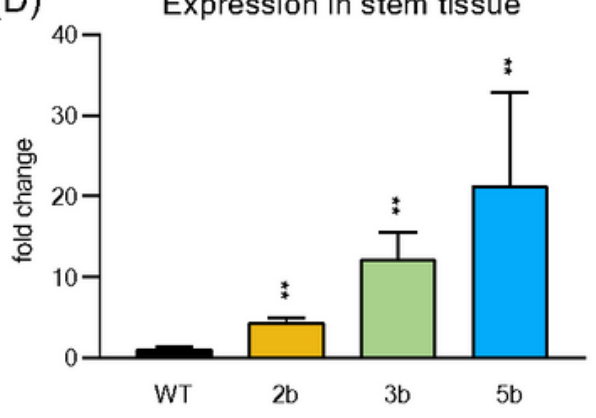

(F) Esterase activity in stem tissue

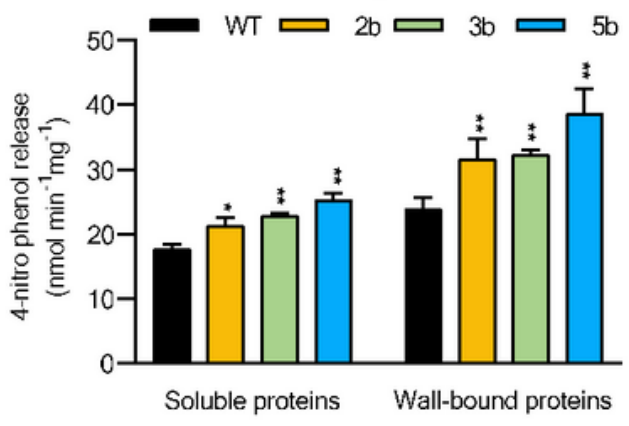

(H) Cell wall acetyl content in stem

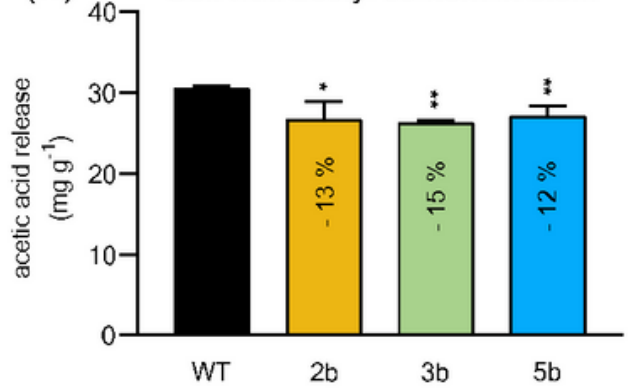

\section{Figure 4}

Evaluation of three independent transgenic lines of Arabidopsis overexpressing 35S::AtAXE1. A Expression data of AtAXE1 gene in Arabidopsis plant from eFP browser (http://bar.utoronto.ca/). B Picture of wild-type and three independent transgenic lines. Expression of AtAXE1 gene in (C) leaf tissue, 
and (D) stem tissue of transgenic lines by RT-PCR. Esterase activity in extracted soluble and wall bound protein fractions incubated with 4-nitrophenyl acetate and released product 4-nitrophenol was analysed by spectrophotometer at $400 \mathrm{~nm}(\mathrm{E})$ in leaf tissue, and (F) in stem tissue. Wild-type and transgenic Arabidopsis $(\mathrm{G})$ leaf, and $(\mathrm{H})$ stem alcohol insoluble residues saponified and acetyl content was analysed by acetic acid kit (K-ACET) from Megazyme. Data represents mean $\pm S E, n=3-4$ biological replicates, Student's t-test at ${ }^{*} \mathrm{p} \leq 0.05,{ }^{*} \mathrm{p} \leq 0.1$.

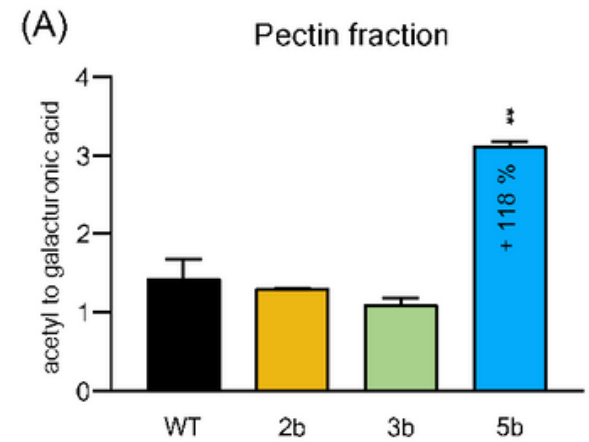

(C)

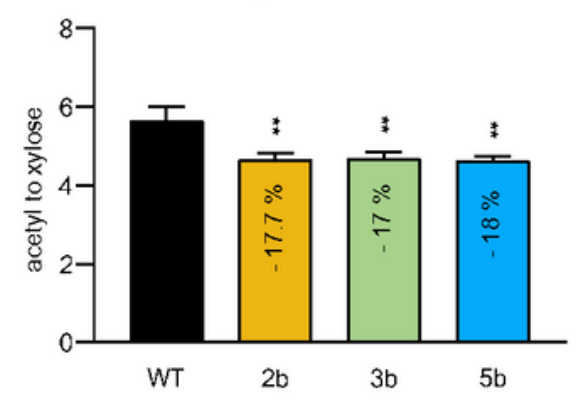

(E)

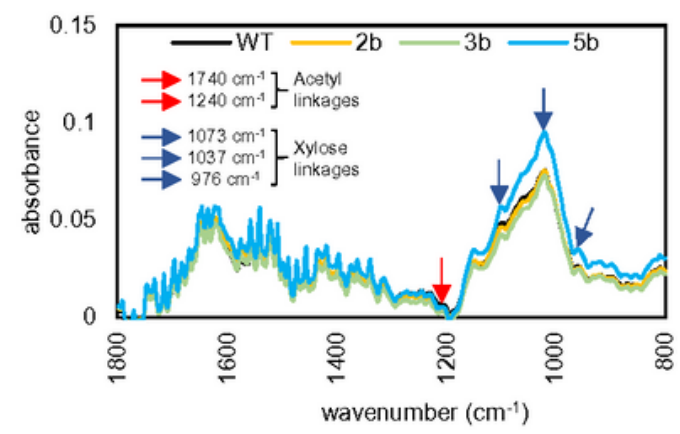

(G)

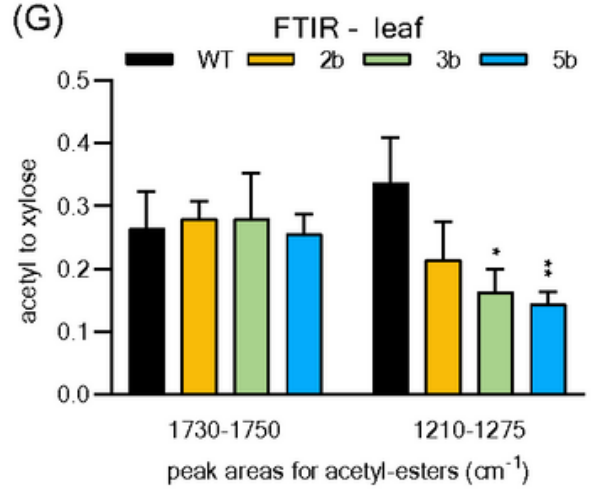

(B) Xylan and xyloglucan rich fraction

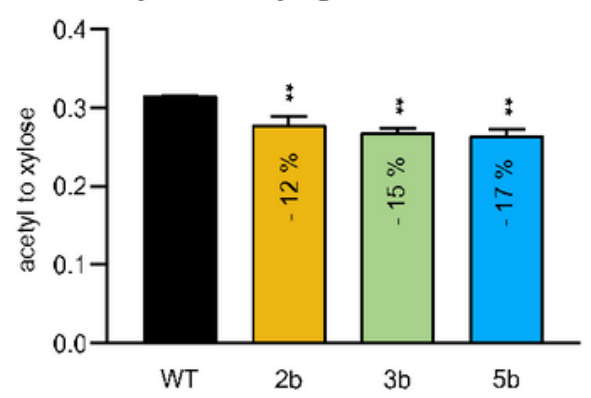

(D) DMSO extracted xylan fraction

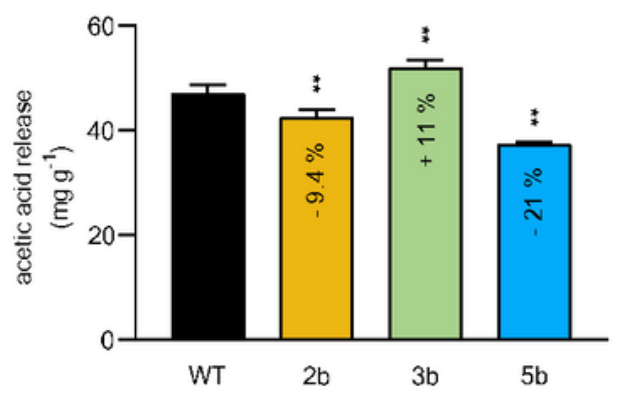

(F)
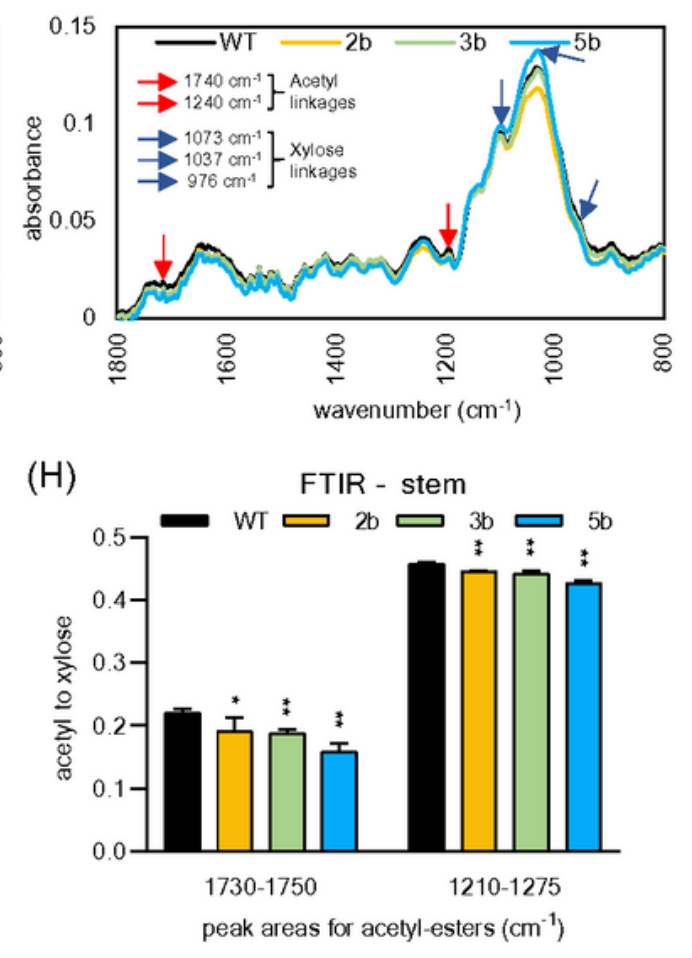

Figure 5 
Sequential extraction of different cell wall polymers and acetyl content analysis in (A) pectin fraction, (B) combined xyloglucan and xylan-rich fraction, (C) xylan-rich fraction, and (D) DMSO extracted xylan fraction. Fourier transform infrared (FTIR) spectroscopy was performed in (E) leaf, and (F) stem tissue of wild type and transgenic Arabidopsis plant, to detect different bonds in cell wall material. The red arrow represents the significant decrease in acetyl-ester linkages. The ratio of wavenumbers corresponding acetyl-ester to xylose peaks in $(\mathrm{G})$ leaf, and $(\mathrm{H})$ stem. Data represents mean $\pm S E, n=3-4$ biological replicates, Student's t-test at ${ }^{* *} p \leq 0.05,{ }^{*} p \leq 0.1$.

(A)
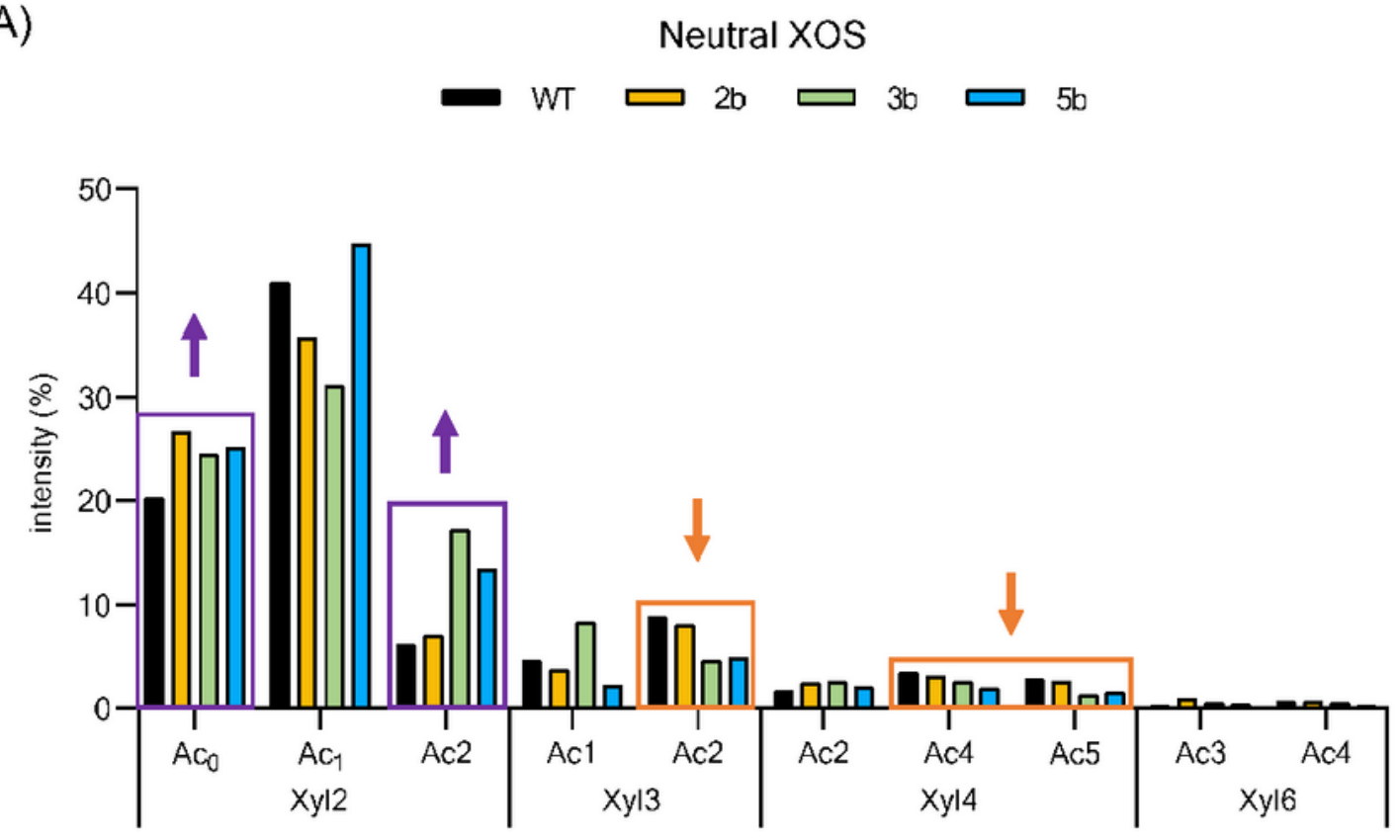

(B)

Acidic XOS

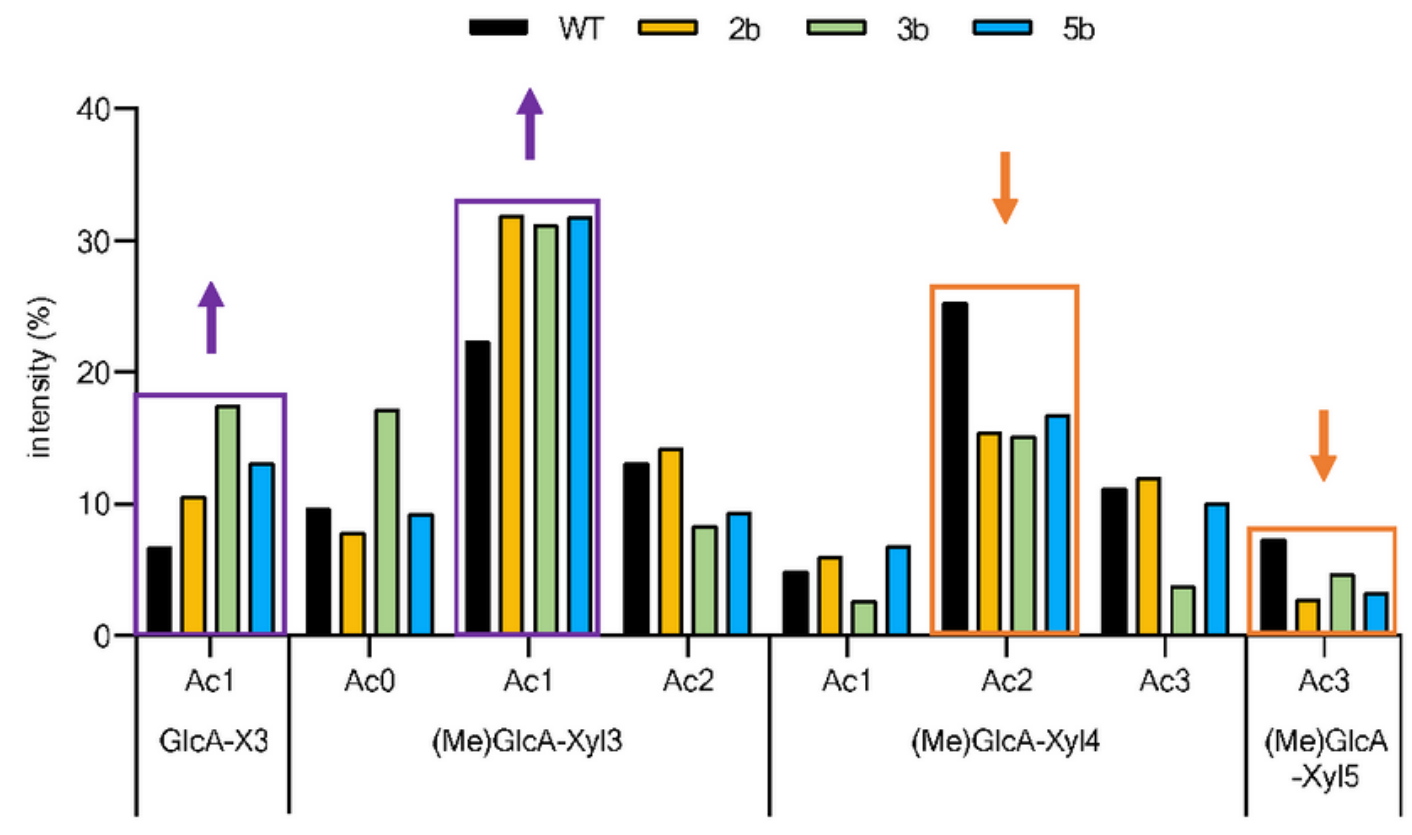


Figure 6

Xylan oligosaccharide analysis in (A) neutral XOS, (B) acidic XOS, released by GH11 $\beta$-endo-xylanase and analyzed by MALDI. Arrows indicate trend of XOS release. Showing this data from one of experiment which is repeated twice.

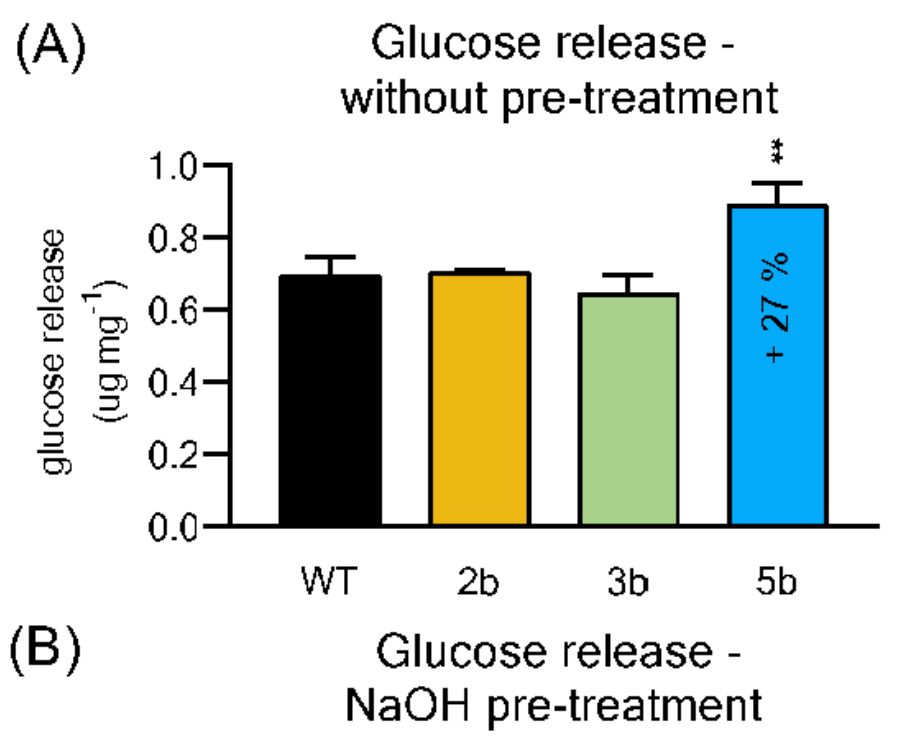
(D)
Cellulose content

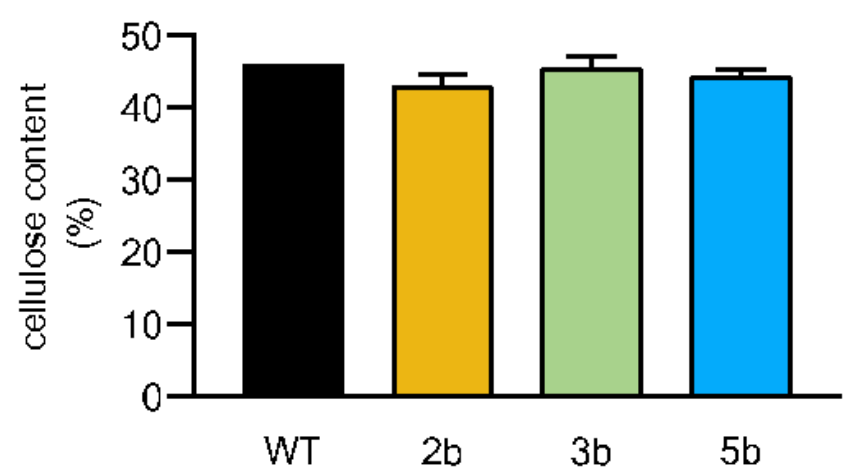

(E)

Xylose content
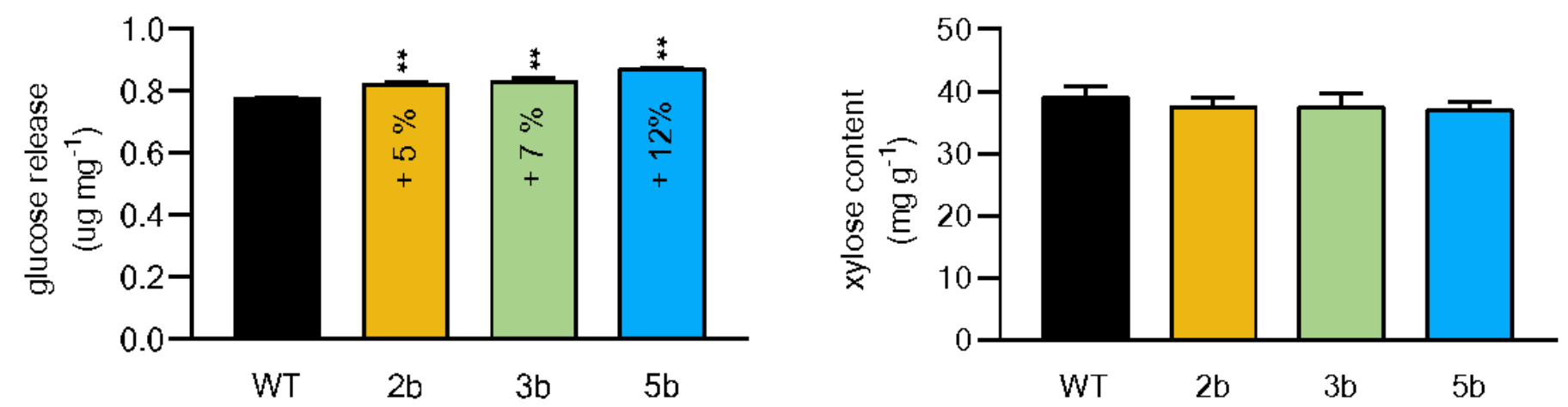

(C)

Glucose release xylanase pre-treatment

(F)

Lignin content
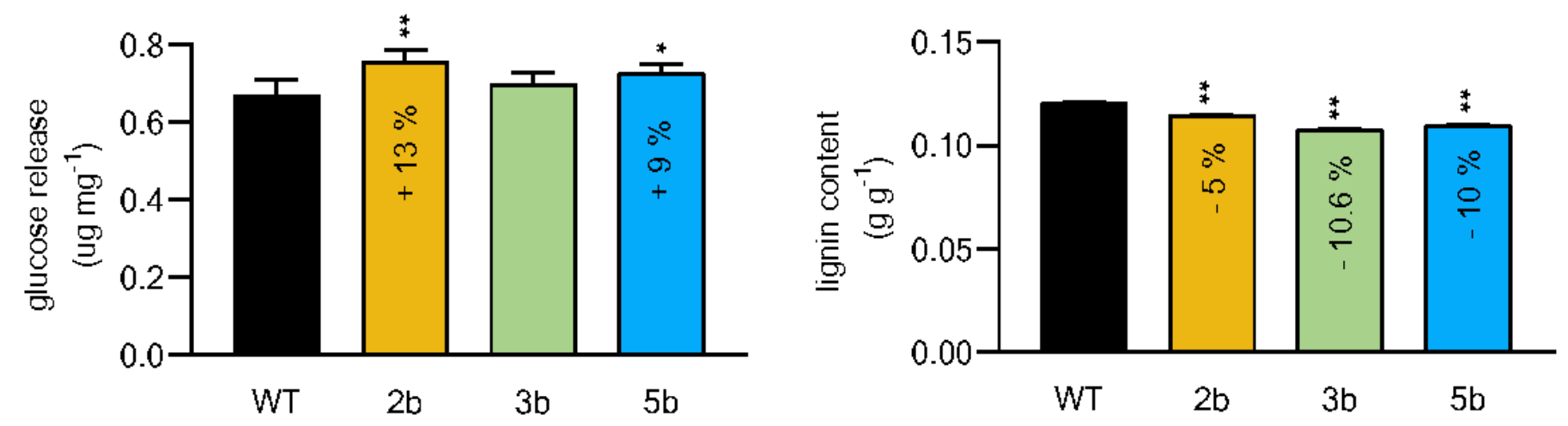

Figure 7 
Improved saccharification of stem lignocellulose in overexpressing 35S::AtAXE1 Arabidopsis transgenic lines. Graphs showing glucose release after enzymatic hydrolysis (A) without pre-treatment, (B) after $\mathrm{NaOH}$ pre-treatment, and (C) with pre-treatment by xylanase. Analysis of major cell wall components in WT and transgenic line stem powder (D) cellulose, (E) xylose (Megazyme D-Xylose Kit), and (F) lignin. Data represents mean $\pm S E, n=3-4$ biological replicates, Student's t-test at ${ }^{*} p \leq 0.05, * p \leq 0.1$

\section{Supplementary Files}

This is a list of supplementary files associated with this preprint. Click to download.

- SupplementaryFiguresPMB.pptx

- SupplementarytablePMB.xlsx 